Review Article

\title{
Traditional Chinese Medicine for Postoperative Care following Anterior Cruciate Ligament Reconstruction: A Systematic Review and Meta-Analysis
}

\author{
Hokyung Chang $\left(\mathbb{D},{ }^{1,2}\right.$ Hyungsuk Kim $\left(\mathbb{D},{ }^{1,2}\right.$ Koh-Woon Kim ${ }^{D},{ }^{2}$ Jae-Heung Cho $\mathbb{D}^{1,2}$ \\ Mi-Yeon Song $\mathbb{D}^{2}$, and Won-Seok Chung $\mathbb{D}^{1,2}$ \\ ${ }^{1}$ Department of Rehabilitation Medicine of Korean Medicine, Kyung Hee University Medical Center, Seoul 02447, \\ Republic of Korea \\ ${ }^{2}$ Department of Clinical Korean Medicine, Graduate School, Kyung Hee University, Seoul 02447, Republic of Korea
}

Correspondence should be addressed to Won-Seok Chung; omdluke@khu.ac.kr

Received 23 March 2021; Accepted 19 August 2021; Published 21 September 2021

Academic Editor: Jing-Yu (Benjamin) Tan

Copyright $\odot 2021$ Hokyung Chang et al. This is an open access article distributed under the Creative Commons Attribution License, which permits unrestricted use, distribution, and reproduction in any medium, provided the original work is properly cited.

\begin{abstract}
Objectives. This review verifies the clinical effects of traditional Chinese medicine (TCM) combined with conventional rehabilitation after anterior cruciate ligament reconstruction (ACLR). Methods. MEDLINE/PubMed, EMBASE, CENTRAL, JMAS, CNKI, and seven Korean databases were searched using predetermined strategies. The risk of bias was assessed using Cochrane Collaboration's tool and a meta-analysis was conducted accordingly. Results. Nineteen randomized controlled trials involving 1283 participants were included in this systematic review and meta-analysis. The TCM treatment group showed more significant improvements in pain ( $\mathrm{MD}-0.74,95 \% \mathrm{CI}[-0.93,-0.54]$; I2 $=89 \%)$, range of motion (ROM) (SMD 1.19, 95\% CI [0.78, 1.59]; $\mathrm{I} 2=78 \%$ ), and knee swelling (SMD $-1.72,95 \%$ CI $[-2.38,-1.07]$; I2 = 76\%). The Lysholm score of the TCM treatment group significantly improved (MD 5.62, 95\% CI [3.93, 7.32]; I2 = 84\%) relative to the control group. The IKDC subjective score (MD $3.40,95 \%$ CI $[-0.61,7.41] ; \mathrm{I} 2=97 \%$ ) and the hospital for special surgery (HSS) score did not improve initially (MD 6.79, $95 \%$ CI $[-1.27,14.86] ; \mathrm{I} 2=97 \%)$ but did so during the subgroup analysis. TCM showed a long-term effect on the IKDC subjective score (MD - 0.51, 95\% CI [-1.69, 0.67]; I2 = 30\%). A longer treatment period of 12 weeks showed more improvement (MD 5.96, 95\% CI $[0.69,11.22]$; I2 95\%). Conclusion. TCM can be used as an adjuvant therapy to conventional rehabilitation for relieving pain, improving ROM and oedema, and facilitating better function of the knee joint after ACLR. However, this recommendation should be cautiously applied in clinical practice owing to the low quality of the included studies.
\end{abstract}

\section{Introduction}

The anterior cruciate ligament (ACL), which is important for stabilizing the knee joint, is the most commonly injured ligament in athletes and trauma victims. The annual incidence of isolated ACL tears is 68.6 per 100,000 personyears [1]. Although the appropriate treatment for an ACL injury depends on its severity and the characteristics of the patient, ACL reconstruction (ACLR) is commonly performed. ACLR generally involves arthroscopy using a graft to replace the injured ACL with the patellar, hamstring, or quadriceps tendon.
Successful ACLR requires appropriate physical rehabilitation focusing on muscle strengthening and the enhancement of balance and proprioception of the knee joint to help patients recover their mobility [2]. However, several rehabilitation programs are often interrupted by pain, stiffness, and swelling of the knee joint after ACLR. To date, there is no consensus yet as to what is the most appropriate rehabilitation program for successful recovery after ACLR [3].

Traditional Chinese medicine (TCM) is characterized by a holistic approach to diagnosis, pathophysiology, and treatment based on basic theories, such as the Yin-yang and Qi theories. Major components of TCM include 
acupuncture, herbal medicine, and other physical therapy, such as massages. The effectiveness of East-West integrative medicine, including acupuncture and herbal medicine, for postoperative care after knee surgery has been continuously discussed [4, 5]. A recent meta-analysis suggested that acupuncture can relieve postoperative pain and reduce opioid consumption after total knee arthroplasty (TKA) [6]. According to another systematic review, electroacupuncture (EA) can offer pain relief after TKA [7]. Several studies have verified the effects of TCM on pain after knee surgery, including TKA, open reduction, and internal fixation (OR/IF) of the knee joint. However, there has been no separate systematic review of the effects of TCM during postoperative care of ACLR. In addition, current systematic reviews involve neuromuscular electrical stimulation (NMES), continuous passive motion therapy (CPM), cryotherapy, and homeopathic arnica therapy, instead of TCM [8]. Therefore, we conducted a systematic review focusing on the effects of TCM on pain after ACLR.

This review assesses the clinical effects of TCM combined with conventional rehabilitation used for postoperative care of ACLR compared with conventional rehabilitation alone. The main objective was to verify the effects of TCM on pain after ACLR. The secondary objective was to reveal the effects of TCM on the range of motion (ROM), the comprehensive evaluation, and the swelling of the knee joint after ACLR. The comprehensive evaluation involved the use of various scales to assess symptoms and function of the knee joint after ACLR.

\section{Methods}

The protocol of this systematic review has been registered with the Open Science Framework (osf.io/zy2w8), and it follows the Preferred Reporting Items for Systematic Reviews and Meta-Analysis (PRISMA) guidelines [9]. The protocol of the current review has been published elsewhere [10].

\subsection{Criteria for Consideration of Studies for This Review}

2.1.1. Types of Studies. This review included only prospective randomized controlled trials (RCTs) on the effects of TCM after ACLR. Nonrandomized controlled trials, retrospective chart reviews, observational studies, and case studies were excluded. There was no language restriction for the studies.

2.1.2. Types of Participants. Patients who were treated with TCM after ACLR were included, and there were no restrictions in age, sex, and the type of procedure or grafts used during reconstruction surgery. The studies involving patients who had undergone other surgeries of the knee joint or suffered from severe comorbidities and complications after surgery were excluded.

2.1.3. Types of Interventions. This study defined various physiotherapy interventions, patient education, and pharmacological treatments as standards of "conventional rehabilitation (CR)" during postoperative care following ACLR in clinical practice. Pharmacological treatments included various analgesics administered orally or through intravenous injections. Physiotherapy included rehabilitation programs such as mobilization of the knee joint using $\mathrm{CPM}$, physical exercise, and skin electrical stimulation treatment.

Experimental Group Intervention. For the experimental group, the intervention had to include TCM treatments. In this review, we defined TCM as interventions including either acupuncture or herbal medicine. The combinations of acupuncture or herbal medicine and other TCM treatments, such as moxibustion, fuming-washing therapy, fumigation, and massaging along the meridian, were permitted accordingly. The combination of TCM and conventional rehabilitation was also permitted if the same treatments were provided to the control group.

With respect to acupuncture, various types of needling, provided that they punctured the skin, as well as other stimulations of the needle, such as electric or heat, were also included in this study. Modalities, which did not involve penetration, including acupressure and laser acupuncture, were not considered acupuncture.

For herbal medicine, all the orally administered forms were included as part of the study. Combinations of two or more types of herbal medicine were also included accordingly. There were no restrictions on the composition, intake dosage or frequency, and application duration.

Control Group Intervention. The control groups were treated with conventional rehabilitation (i.e. physiotherapy, analgesics, patient education). They were subject to no other restrictions under the assumption that the same treatments were applied to the intervention group. If a study involved a control group of patients who were treated with TCM therapy, it was excluded because this review was designed to compare TCM and other modalities.

2.1.4. Types of Outcome Measures. Primary Outcomes. The primary outcomes included all the indicators for evaluating pain (i.e. visual analogue scale (VAS) and numerical rating scale (NRS)). If the pain scores during rest and activity were presented, those related to activity were selected because physiotherapy after ACLR included several activities for promoting muscle strength and flexibility in clinical practice.

Secondary Outcomes. The secondary outcomes included the ROM of the knee joint and indicators for evaluating symptoms, function of the knee joint (i.e. Lysholm score, International Knee Documentation Committee 2000 subjective knee form (IKDC Subjective score), Hospital for Special Surgery (HSS) score), and swelling of the knee joint taken from the knee circumference, after ACLR.

2.2. Search Methods for the Identification of Studies. The Cochrane Central Register of Controlled Trials (CENTRAL), MEDLINE/PubMed, and EMBASE were searched for articles. One Chinese database (Chinese National Knowledge Infrastructure; CNKI), one Japanese database (Japan 
Medical Abstracts Society; JMAS), and seven Korean databases (Korean National Assembly Digital Library, Korean Association of Medical Journal Editors, Oriental Medicine Advanced Searching Integrated System, Korean Studies Information Service System, National Digital Science Library, Database Periodical Information Academic, and Korean Traditional Knowledge Portal) were systematically searched for studies published from their inceptions to June 2020 by two reviewers (H. C., H. K.). The search process was based on specific keywords from four broad concepts of interest: (1) "anterior cruciate ligament reconstruction," (2) "acupuncture," (3) "Chinese herbal medicine," and (4) "randomized controlled trial." The complete search strategy for the CENTRAL, MEDLINE/PubMed, EMBASE, and CNKI databases is presented in the Appendix.

To find relevant literature omitted from the search above, the references of these papers were screened by their titles and abstracts. The World Health Organization International Clinical Trials Registry Platform (ICTRP) was explored for unpublished trials. Furthermore, literature that could not be searched online (i.e. hard copy) were manually searched. We also contacted the researchers of ongoing studies to verify information when required.

\subsection{Data Collection and Analysis}

2.3.1. Selection of Studies. Using predetermined strategies, two reviewers (H. C., H. K.) independently searched the aforementioned databases. For database articles, ambiguous literature, and manually searched hard copies, the reviewers performed primary screening by applying predetermined inclusion and exclusion criteria after reading the titles and the abstracts. The predetermined criteria were applied to the full-texts of studies to select the RCTs for our systematic review. When consensus on the selection process could not be reached, a third reviewer (W. C.) made the final decision about including or excluding ambiguous studies.

2.3.2. Data Extraction and Management. The reviewers extracted information from each article through a full-text review of the finally selected articles. When the collected data were incomplete or unclear, the arbiter contacted the authors of the original articles to request additional data or further explanation. We obtained data on the demographics of the sample, onset of ACL injury, date from ACLR to initial TCM treatment, details of intervention in experimental groups, details of intervention in control groups, types of outcome measurements, evaluation time points after surgery, and adverse events. The reviewers made final decisions on any issues following consultation with an arbiter (W. C.) when consensus could not be reached.

2.3.3. Risk of Bias Assessment. Two independent reviewers evaluated the risk of bias of the included studies using the Cochrane Collaboration tool (risk of bias, ROB) to assess the quality of each RCT. Seven domains were used for the assessment: random sequence generation, allocation concealment, blinding of participants and personnel, blinding of outcome assessment, incomplete outcome data, selective outcome reporting, and other sources of bias. Each domain was rated as high risk, low risk, or unclear risk. When a consensus on the assessment of the ROB could not be reached through consultations, a third reviewer (W. C.) was consulted to make a final decision.

2.3.4. Quantitative Data Synthesis. The mean differences (MDs) with 95\% confidence intervals (CIs) were used for the analysis of continuous data. Weighted mean differences (WMDs) were adopted when the same scale was used, whereas standardized mean differences (SMDs) were used if different indicators were used to measure certain outcomes of the included studies.

When a study reported multiple group comparisons, only data from the treatment group that received more intensive conventional intervention were included in the analysis. For studies with a crossover research design, data from the first sessions were obtained accordingly.

We conducted a meta-analysis to estimate the differences between groups in the primary and secondary outcomes using the Cochrane Collaboration software (Review Manager Software Version 5.3). Depending on the level of heterogeneity among the included studies, we applied a fixed-effects model or a random-effects model. When heterogeneity was relatively high $\left(\mathrm{I}^{2}>50 \%\right)$, a random-effects model with $95 \%$ CI was used to analyze the pooled effect estimates [11]. Heterogeneity was assessed in the following three ways according to the guidelines of the Cochrane Handbook for Systematic Reviews of Interventions: (1) a visual check of the forest plot, (2) using a heterogeneity $\chi^{2}$ test, and (3) using Higgins $\mathrm{I}^{2}$ statistic. In interpreting the heterogeneity $\chi^{2}$ test, a significance level of $p<0.10$ was used to represent meaningful heterogeneity. A value of Cochrane's Higgins $\mathrm{I}^{2}$ greater than $75 \%$ represented considerable heterogeneity. A subgroup analysis was conducted to identify the reasons for heterogeneity when considerable heterogeneity was detected. If meaningful heterogeneity could not be explained by subgroup analysis, we did not conduct a meta-analysis.

2.3.5. Subgroup Analysis. When it was necessary to explain the considerable heterogeneity of the included studies, we conducted a subgroup analysis based on the following: (1) the type of TCM treatments (i.e. acupuncture alone, herbal medicine alone, acupuncture plus herbal medicine, acupuncture plus more than one other TCM treatment, herbal medicine plus more than one other TCM treatment, acupuncture plus herbal medicine with more than one other TCM treatment), (2) the time points of evaluation after reconstruction surgery ( $<2$ weeks, $2-4$ weeks, $4-8$ weeks, 8-12 weeks, $12-16$ weeks, 16 weeks- 1 year, and more than a year), and (3) duration of treatment ( $<2$ weeks, $2-4$ weeks, 4-7 weeks, 7-8 weeks, more than 12 weeks). The time points of evaluation were established according to the stage of 
rehabilitation after ACLR [12] because symptoms such as pain, ROM, oedema, and dysfunction of the knee joint differ with the stage of rehabilitation.

\section{Results}

3.1. Included Studies. A total of 254 articles were retrieved from the online search. Initially, two of the authors (H.C., H.K.) screened the articles, and 74 records were removed because of duplicates. The title and abstracts of the remaining articles were further examined for eligibility, and 151 records were eliminated for several reasons: not being about ACLR, interventions without acupuncture nor herbal medicine, acupuncture treatment in the control group, no outcomes of interest, not RCTs, animal studies, ongoing research without available results, and inaccessible full texts. Subsequently, the full-text articles were assessed for eligibility, and 10 records were eliminated for various reasons: interventions without acupuncture nor herbal medicine, acupuncture treatment in the control group, herbal medicine treatment in the control group, not RCTs, no outcome of interest, and insufficient outcome data which were not obtained even though we had contacted the author(s) of the original study. Finally, 235 articles were excluded, and 19 RCTs involving a total of 1283 patients were included for analysis. The reasons for exclusion and the selection flow are presented in Figure 1.

3.2. Characteristics of the Studies. The characteristics of the included studies are presented in Table 1. The included RCTs were published between 2006 and 2020, with 18 of them published in Chinese [13-30] and one of them published in English [31]. Of the included studies, 18 were implemented in China [13-30] and one in Spain [31]. The sample sizes of the studies ranged from 25 to 160 . All the patients in the studies had undergone ACLR for ACL injury, and their mean ages were between 25 and 38. The onset of ACL injury varied from 4 hours to 3 years, and the time of initiation of intervention after reconstruction surgery ranged from immediately after surgery to approximately 4 months after surgery.

Various TCM treatments were applied to the experimental group. Seven studies adopted only acupuncture as TCM treatment, including manual acupuncture alone $[15,22,30,31]$, manual acupuncture combined with warm needling [23], or manual acupuncture combined with electroacupuncture $[16,21]$. One study used manual acupuncture and electroacupuncture combined with fumingwashing therapy and massaging along the meridian [14]. In three studies, patients were treated with herbal medicine alone $[20,28,29]$, whereas in the other three, patients were treated with herbal medicine combined with fumingwashing therapy [24] or massaging along the meridian $[17,18]$. In five studies, acupuncture and herbal medicine were adopted as the main TCM treatments. Of them, three used electroacupuncture and herbal medicine combined with massaging along the meridian [19] or fuming-washing therapy $[25,26]$, while one study used both manual acupuncture and electroacupuncture combined with herbal medicine and fuming-washing therapy [13]. The other one used manual acupuncture and herbal medicine combined with fuming-washing therapy and massaging along the meridian [27].

The durations of the treatments in the experimental groups ranged from a day [31] to one year [20]. The details of the acupuncture treatments are presented in Table 2. Acupuncture was performed from the western medical point of view targeting the vastus medialis muscle trigger point $(\operatorname{TrP})$ in only one study [31], whereas the others used specific acupuncture points near the affected knee joint based on the TCM theory. The top ten frequently used acupuncture points were SP10, ST36, GB34, SP9, SP6, ST34, BL57, ST32, ST35, and BL39, all of which were near the muscles of the affected knee joints. The frequently used acupuncture points are organized in Table 3. Acupuncture treatments were applied for durations between 15 minutes and 30 minutes accordingly.

The dosage forms and frequencies of the herbal medicine treatments varied with each study, and the details are presented in Table 4. The durations of the herbal medicine treatments ranged from three weeks [13] to one year [20]. The most frequently used Chinese medicinal herb was Angelicae Gigantis Radix. Two studies [25, 27] did not provide any information about the basic components of herbal medicine. The frequently used Chinese medicinal herbs are organized in Table 5.

All the patients in the control groups had undergone conventional rehabilitation, including physiotherapy, patient education, and pharmacological treatments. The same treatments were applied to the experimental group.

Fifteen studies used the VAS as the main indicator for measuring pain after ACLR [13-19, 21-26, 31]. One study did not present standard deviation data and was thus excluded from the analysis because we could not receive any data from the author of the original study [17]. In three studies $[19,25,26]$, the VAS scores obtained during rest and activity were presented, and the VAS score during activity was included in the analysis as planned. Ten studies presented the ROM of the affected knee joint. Four studies [14, 18, 23, 26] measured the active ROM, and one study [15] measured the passive ROM of the affected knee joint. The other four studies $[19,24,25,31]$ did not mention specific measurement methods Fourteen studies [13, 14, 17-20, 22-29] reported the Lysholm scores, six studies $[17,18,20,25,26,28]$ reported the IKDC subjective scores, and three studies [21, 25, 30] presented the HSS scores, which are comprehensive indicators for evaluating the symptoms and function of the knee joint after ACLR. Four studies $[14,15,18,24]$ measured the circumferences of the affected knee joint near the patella bone to evaluate knee joint swelling.

Several studies reported adverse events after TCM treatment. One study [31] reported that three patients suffered hemorrhages larger than $4 \mathrm{~cm}^{2}$, which were not significantly different in the experimental and control groups $(p=0.073)$. Another study [25] reported that three 


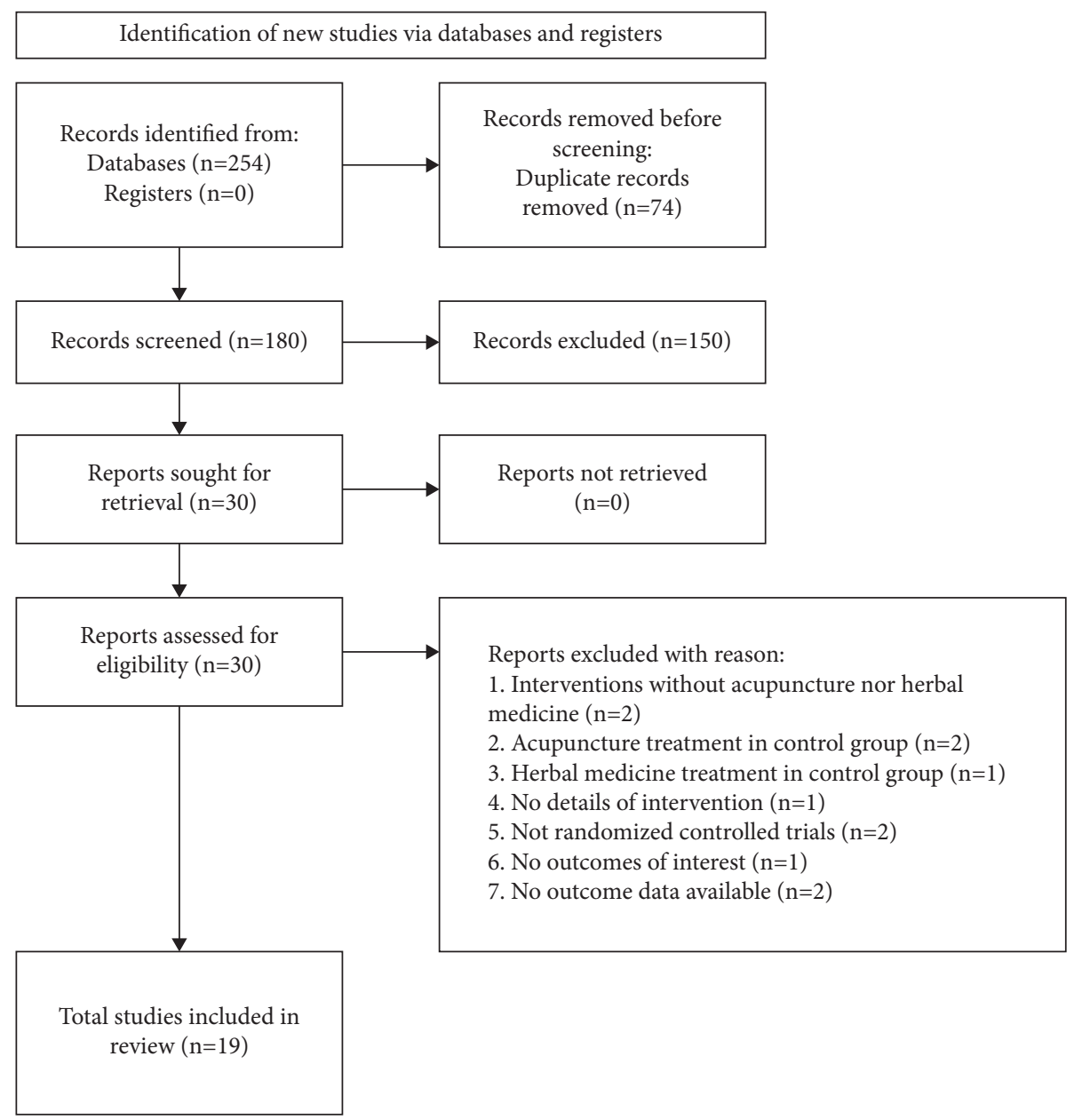

FIgURE 1: Flow diagram of the study selection process.

patients suffered from diarrhea, which resolved spontaneously after taking herbal medicine. One study [16] compared adverse events such as headache, nausea, vomiting, abdominal pain, diarrhea, leg oedema, and dizziness in the electroacupuncture and the analgesics groups. Of 20 patients in the control group, three complained of nausea, vomiting, and abdominal pain after taking analgesics, whereas only one patient of 20 in the experimental group complained of dizziness after acupuncture treatment. The difference was statistically significant $(p=0.004)$.

3.3. Risk of Bias in Included Studies. Of the studies included in this review, $10[14-16,20,22-24,28,30,31]$ used the appropriate randomization method, while nine studies [13, 17-19, 21, 25-27, 29] did not provide specific descriptions about their randomization methods (Figure 2). Two studies $[20,31]$ adequately carried out allocation concealment using opaque closed-letter envelopes, while the other studies did not describe their approaches to allocation concealment in detail. No studies were evaluated as low-risk based on participant and personnel blinding because it was difficult to exclude performance bias in administering the TCM treatments. Fifteen studies [13-15, 17-24, 27-30], which involved single-blinded participants, were evaluated as high risk. The other studies did not indicate participant blinding. Only one study [31] provided clarifications on the blinding methodologies and assessment of outcomes. The other studies did not clarify the specific methods used to prevent detection bias. Three studies $[20,28,31]$ showed dropouts within the domain of incomplete outcome data, but this was considered insignificant because the dropout rates of the groups were very low and similar between experimental groups and control groups, respectively. The other two studies $[18,22]$ did not indicate dropout or withdrawal. In seventeen studies, selective reporting was not decided and thus reporting was ambiguous. One study [31] published its protocol, and all of the prespecified outcomes were reported in the results. Another study [26] reported different evaluation time points in the methods and the results sections. Other forms of bias were evaluated based on whether there was no significant difference between the general characteristics of the patients in the experimental and control groups before treatment. All the included studies, except one [28], clarified that there was no significant difference between the general characteristics of the groups.

3.4. Effects of Interventions. Nineteen studies [13-31] involving a total of 1283 participants were included in the meta-analysis. The meta-analysis was based on six outcomes: 


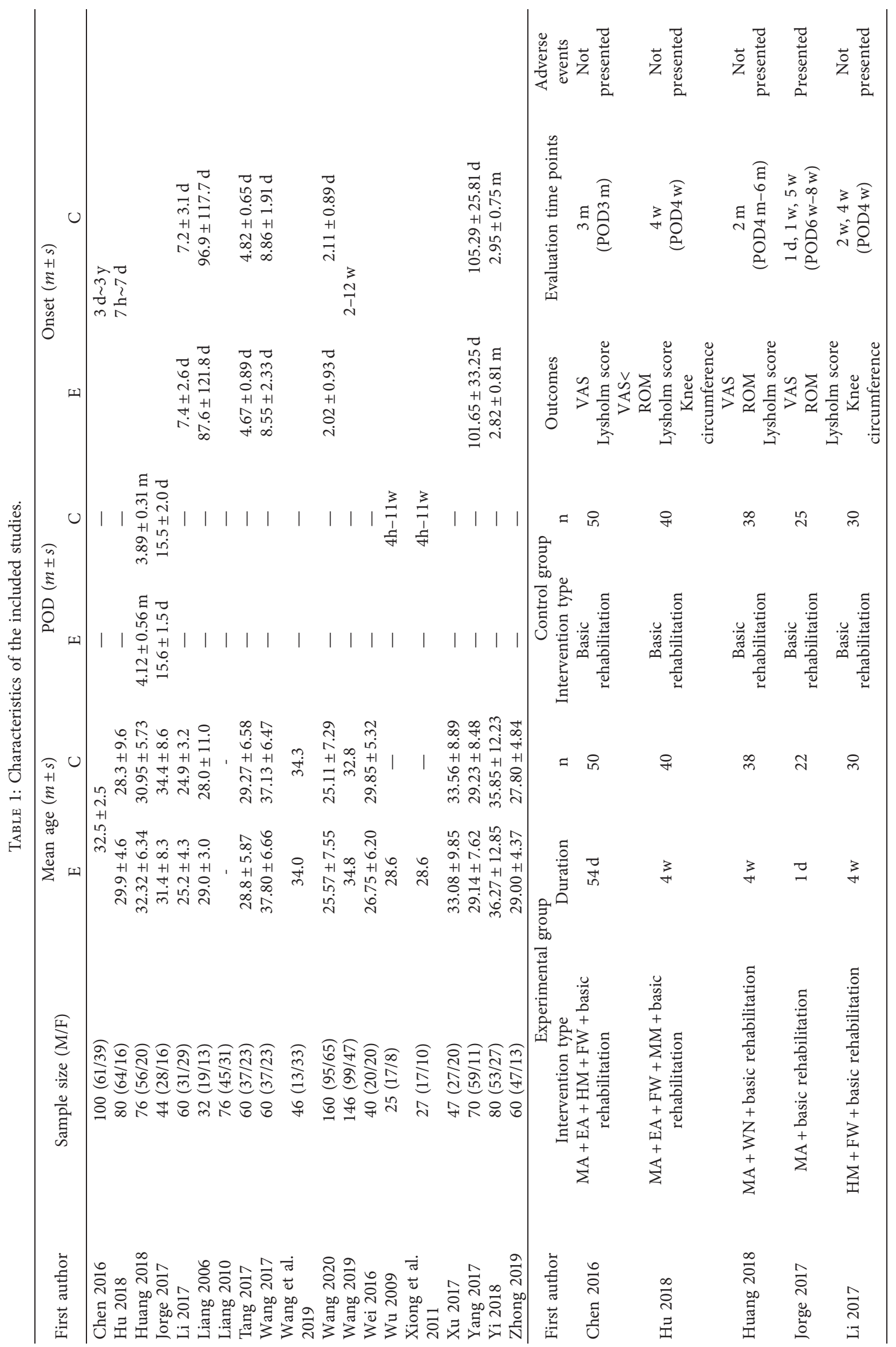




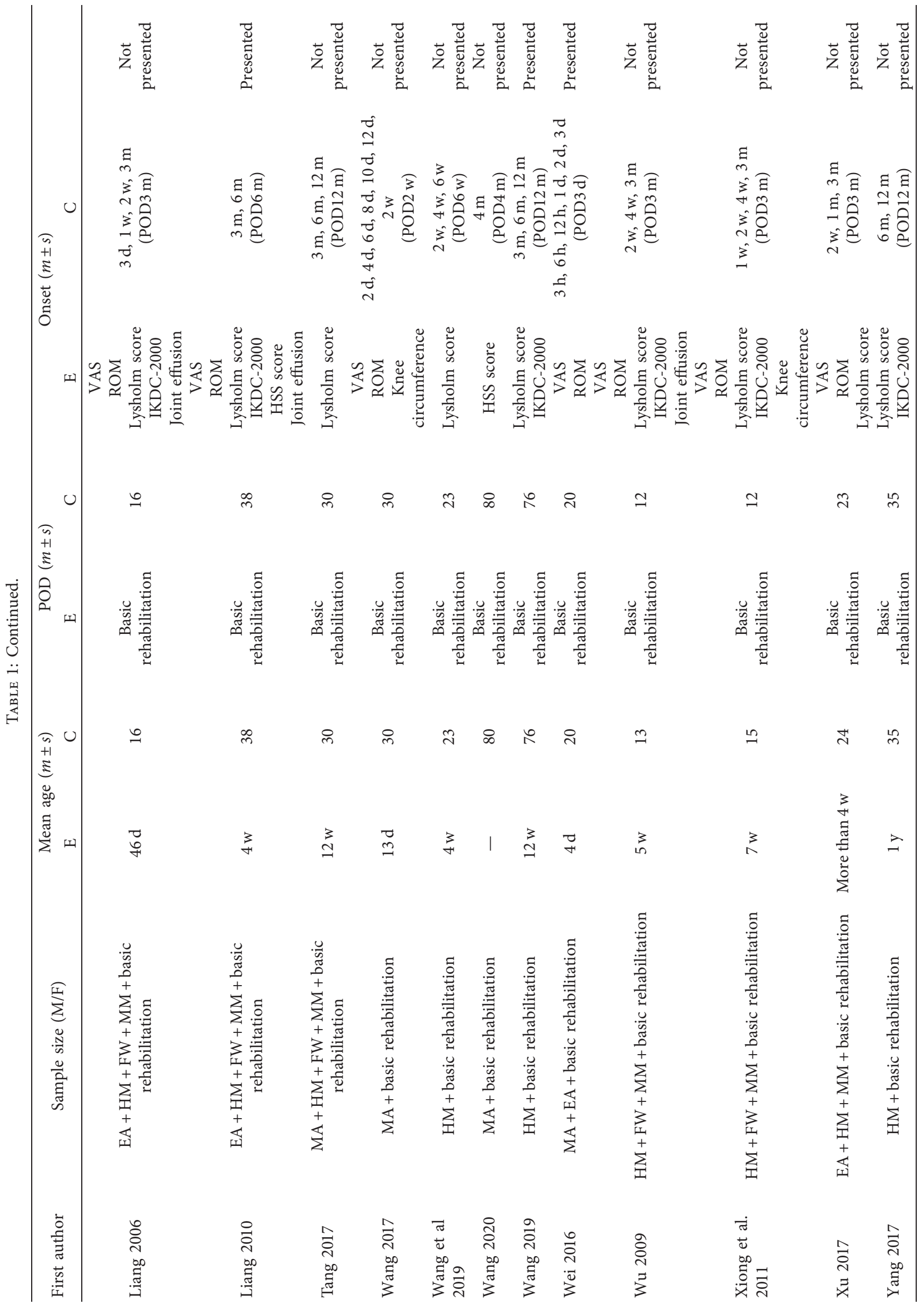




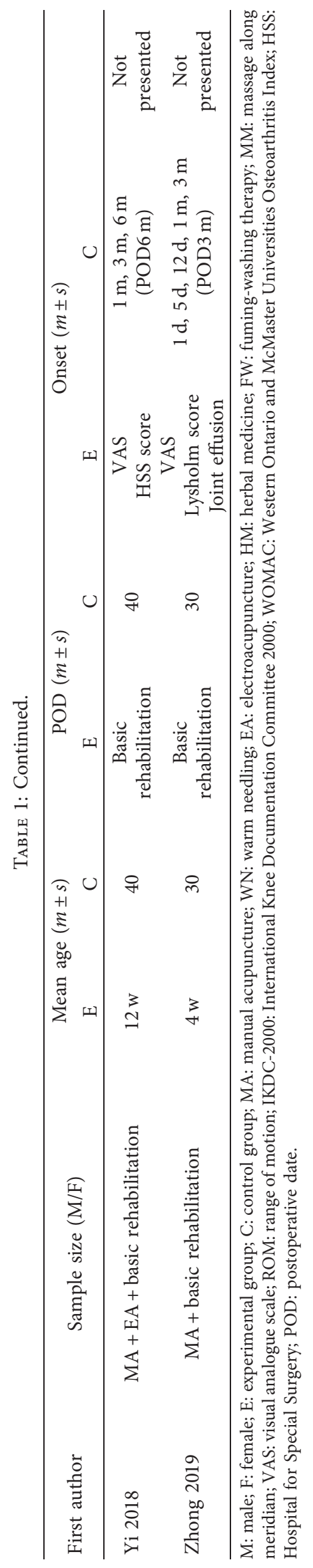


TABLE 2: Details of acupuncture treatment.

\begin{tabular}{|c|c|c|c|c|}
\hline \multirow[b]{2}{*}{ First author } & \multirow{2}{*}{$\begin{array}{l}\text { Acupuncture } \\
\text { rationale }\end{array}$} & \multicolumn{3}{|c|}{ Details of needling } \\
\hline & & $\begin{array}{c}\text { Type of } \\
\text { acupuncture }\end{array}$ & Acupuncture points & $\begin{array}{l}\text { Needling duration (ES } \\
\text { frequency) }\end{array}$ \\
\hline \multirow{2}{*}{ Chen 2016} & \multirow{2}{*}{ TCM } & MA & GB31, GB34, ST36, ST40 & $15 \mathrm{~min}$ \\
\hline & & EA & BL57, GB31, GB34, SP6, SP10, ST32, ST36, ST40 & $15 \min (80 \mathrm{~Hz})$ \\
\hline \multirow{2}{*}{ Hu 2018} & \multirow{2}{*}{ TCM } & MA & BL57, GB34, SP9, SP10, ST34, ST35, ST36 & $30 \mathrm{~min}$ \\
\hline & & EA & BL57, GB34, SP9, SP10, ST34, ST35, ST36 & $30 \min$ (n.r.) \\
\hline Huang 2018 & TCM & MA & \multirow{5}{*}{$\begin{array}{c}\text { LR3, SP9, SP10, ST32, ST34, ST35, ST36, EX-LE4 } \\
\text { Vastus medialis TrP } \\
\text { GB34, SP9, ST36 } \\
\text { n.r. } \\
\text { BL39, BL40, BL57, GB33, GB34, LR7, SP6, SP10, } \\
\text { ST34, ST35, ST36 }\end{array}$} & $30 \mathrm{~min}$ \\
\hline Jorge 2017 & Western medical & MA & & $1-2 \mathrm{~min}$ \\
\hline Liang 2006 & TCM & EA & & n.r. $(80 \mathrm{~Hz})$ \\
\hline Liang 2010 & TCM & EA & & n.r. (n.r.) \\
\hline Tang 2017 & TCM & MA & & n.r. \\
\hline Wang 2017 & TCM & MA & SP6, SP9, SP10, ST32, ST34, ST36 & $30 \mathrm{~min}$ \\
\hline Wang 2020 & TCM & MA & $\begin{array}{l}\text { GB34, GV20, KI3, LI4, LR3, SP6, SP9, SP10, ST34, } \\
\text { EX-LE2, zuyundongqu }\end{array}$ & $30 \mathrm{~min}$ \\
\hline \multirow{2}{*}{ Wei 2016} & \multirow{2}{*}{ TCM } & MA & LR6 & $30 \mathrm{~min}$ \\
\hline & & EA & LR6 & $30 \min (100-1000 \mathrm{~Hz})$ \\
\hline Xu 2017 & TCM & EA & BL57, GB34, SP6, SP9, SP10, ST36, ST40 & n.r. \\
\hline \multirow{2}{*}{ Yi 2018} & \multirow{2}{*}{ TCM } & MA & $\begin{array}{c}\text { BL39, BL40, BL57, GB33, GB34, LR7, SP6, SP9, SP10, } \\
\text { ST34, ST35, ST36 }\end{array}$ & $30 \mathrm{~min}$ \\
\hline & & EA & $\begin{array}{c}\text { BL39, BL40, BL57, GB33, GB34, LR7, SP6, SP9, SP10, } \\
\text { ST34, ST35, ST36 }\end{array}$ & $30 \min$ (n.r.) \\
\hline Zhong 2019 & TCM & MA & GB34, SP6, SP9, SP10, ST32, ST34, ST36 & $20 \mathrm{~min}$ \\
\hline
\end{tabular}

TCM: traditional Chinese medicine; MA: manual acupuncture; EA: electroacupuncture; ES: electrical stimulation; n.r: not reported.

TABLE 3: Frequently used acupuncture points.

\begin{tabular}{lccccc}
\hline Number of times used & 9 & 8 & 7 & 5 & 4 \\
\hline Acupuncture point & SP10, ST36 & GB34, SP9 & SP6, ST34 & BL57 & ST32, ST35 \\
\hline
\end{tabular}

the VAS score for pain evaluation, ROM for measuring joint mobility, Lysholm score, IKDC subjective score, HSS score for the comprehensive evaluation of the symptoms and function of the knee joint, and knee circumference for measuring knee swelling. Because the methods for measuring ROM and the knee circumference were different in each study, SMD was employed for the meta-analysis. A subgroup analysis was conducted for all the outcomes because considerable heterogeneities were revealed in the analysis of the pooled effect.

3.4.1. Primary Outcomes. In the meta-analysis of thirteen studies $[13-16,18,19,21-26,31]$ involving 780 subjects, the TCM group showed more significant improvement in the VAS score after TCM treatment than the control group (MD $-0.74,95 \%$ CI $[-0.93,-0.54]$; I2 $=89 \%$; Figure 3$)$. As planned, the subgroup analysis was based on the types of TCM treatments, evaluation time points after surgery, and durations of treatment. All the TCM treatment types, including the acupuncture (MD $-0.71,95 \% \mathrm{CI}-0.92$ to -0.49 , and I2 48\%), acupuncture plus other TCM (MD -1.09, 95\% CI $[-1.17,-1.01])$, herbal medicine plus other TCM (MD $-1.01,95 \%$ CI $[-1.14,-0.89]$; I2 = 0\%), and acupuncture plus herbal medicine combined with other TCM (MD -0.50, 95\% CI $[-0.95,-0.06]$; I2 = 93\%) showed significantly better pain relief after surgery than the control group (Figure 3). For the evaluation time points after surgery, all the periods between $0-2$ weeks after surgery $(\mathrm{MD}-0.50,95 \% \mathrm{CI}[-0.73,-0.27]$; $\mathrm{I} 2=0 \%), 2-4$ weeks after surgery $(\mathrm{MD}-1.28,95 \% \mathrm{CI}[-1.76$, $-0.80]), 4-8$ weeks after surgery (MD $-1.00,95 \%$ CI $[-1.18$ to $-0.82] ; \mathrm{I} 2=40 \%), 12-16$ weeks after surgery $(\mathrm{MD}-0.62$, $95 \%$ CI $[-1.04,-0.20] ; \mathrm{I} 2=95 \%)$, and more than 16 weeks after surgery (MD $-0.74,95 \%$ CI $[-0.92,-0.54] ; \mathrm{I} 2=0 \%)$ were associated with significantly better pain relief than the control group (not shown). For treatment duration, TCM was more effective for pain relief than the control regardless of whether the treatment duration was short or long (Figure 4): $0-1$ week (MD $-0.50,95 \%$ CI $[-0.75,-0.24]$; I2 $=0 \%)$, $2-4$ weeks (MD $-0.95,95 \%$ CI $[-1.15,-0.74]$; I2 = 73\%), 7-8 weeks (MD $-0.74,95 \%$ CI $[-1.03,-0.46]$; I2 $=85 \%), 12$ weeks (MD $-0.76,95 \%$ CI $[-1.34,-0.18])$.

3.4.2. Secondary Outcomes. ROM of the Knee Joint. In the meta-analysis of 10 studies involving 540 patients, the TCM group showed significantly better improvement in the knee ROM than the control group (SMD 1.19, 95\% CI $[0.78,1.59]$; $\mathrm{I} 2=78 \%$; Figure 5). In the subgroup analysis, all the TCM treatments were more effective than the control for ROM improvement regardless of the TCM treatment types: acupuncture (SMD 1.15, 95\% CI $[0.45,1.86]$; I2 = 82\%), acupuncture plus other TCM (SMD 1.08, 95\% CI $[0.61,1.55]$ ), herbal medicine plus other TCM (SMD 1.50, 95\% CI [1.02, 
TABLE 4: Details of herbal medicine treatment.

\begin{tabular}{|c|c|c|c|c|}
\hline $\begin{array}{l}\text { First } \\
\text { author }\end{array}$ & Dosage form & $\begin{array}{c}\text { Administration } \\
\text { duration and frequency }\end{array}$ & $\begin{array}{l}\text { Herbal medicine } \\
\text { name }\end{array}$ & Basic components \\
\hline
\end{tabular}

\begin{tabular}{|c|c|c|c|c|}
\hline author & Dosage form & duration and frequency & name & Basic components \\
\hline \multirow{4}{*}{$\begin{array}{l}\text { Chen } \\
2016\end{array}$} & \multirow{4}{*}{ Decoction } & \multirow{4}{*}{$3 \mathrm{w}$, tid } & 1. Xishangyihao-fang & $\begin{array}{l}\text { 1. Achyranthis Radix, Coptidis Rhizoma, Curcumae } \\
\text { Longae Radix, Rhei Rhizoma Preparata Cum Vinum }\end{array}$ \\
\hline & & & 2. Xishangerhao-fang & 2. Angelicae Gigantis Radix, Bletillae Rhizoma, Cyperi \\
\hline & & & & Rhizoma, Coptidis Rhizoma, Persicae Semen \\
\hline & & & $\begin{array}{l}\text { 3. Xishangsanhao- } \\
\text { fang }\end{array}$ & $\begin{array}{l}\text { 3. Astragali Radix, Chaenomelis Fructus, Cibotii } \\
\text { Rhizoma, Codonopsis Pilosulae Radix, Homalomenae } \\
\text { Rhizoma }\end{array}$ \\
\hline
\end{tabular}

1. Achyranthis Radix, Angelicae Gigantis Radix, Araliae

Continentalis Radix, Aucklandiae Radix, Cnidi Fructus, Cnidii Rhizoma, Coicis Semen, Drynariae Rhizoma,

Li $2017 \quad$ Tablet preparation $\quad 4$ w, tid

1. Guxiyi decoction

Glycyrrhizae Radix Et Rhizoma, Eupolyphaga, Mori

Ramulus, Moutan Cortex Radicis, Myrrha, Olibanum, Notoginseng Radix, Paeoniae Radix, Phryma

Leptostachya, Syzygii Flos, Rehmanniae Radix Crudus, Salviae Miltiorrhizae Radix

\begin{tabular}{|c|c|c|c|}
\hline & & & 1. Xishangyihao-fang \\
\hline $\begin{array}{l}\text { Liang } \\
2006\end{array}$ & Decoction & $4 \mathrm{w}$, tid & 2. Xishangerhao-fang \\
\hline
\end{tabular}

1. Achyranthis Radix, Coptidis Rhizoma, Curcumae

Longae Radix, Rhei Rhizoma Preparata Cum Vinum, Scutellariae Radix, Taraxaci Herba

2. Achyranthis Radix, Akebiae Caulis, Angelicae

Gigantis Radix, Bletillae Rhizoma, Coptidis Rhizoma,

Cyperi Rhizoma, Dipsaci Radix, Paeoniae Radix, Persicae Semen

3. Xishangsanhao-

3. Akebiae Caulis, Astragali Radix, Chaenomelis fang

Fructus, Cibotii Rhizoma, Cistanchis Herba, Codonopsis Pilosulae Radix, Cuscutae Semen, Lumbricus, Homalomenae Rhizoma, Visci Herba Et Loranthi Ramulus

\begin{tabular}{lcll}
\hline Liang & Decoction & $4 \mathrm{w}$, tid & $\begin{array}{l}\text { 1. Xishangyihao-fang } \\
2010\end{array}$ \\
\hline Tang 2017 & $\begin{array}{c}\text { Decoction, } \\
\text { powder } \\
\text { preparationgerhao-fang }\end{array}$ & \multirow{2}{*}{$6 \mathrm{w}$, tid } & $\begin{array}{l}\text { 1. XiaoZhong } \\
\text { ZhiTong mixture } \\
\text { 2. Sunshangsan }\end{array}$ \\
\hline
\end{tabular}

Wang
et al. $2019 \quad$ Decoction

1. Angelicae Gigantis Radix, Carthami Flos, Cnidii

et al. 2019

\begin{tabular}{|c|c|c|}
\hline $\begin{array}{l}\text { Wang } \\
2019\end{array}$ & Pill preparation & $12 \mathrm{w}, \mathrm{bid}$ \\
\hline
\end{tabular}

Radix Preparata

1. Acanthopanax Root Bark, Achyranthis Radix, Angelicae Gigantis Radix, Aucklandiae Radix,

Chaenomelis Fructus, Cistanchis Herba, Cnidi Fructus, Cuscutae Semen, Dioscoreae Rhizoma, Ginseng Radix, Olibanum, Poria Sclerotium, Rehmanniae Radix Preparata, Tribuli Fructus

\begin{tabular}{|c|c|c|c|c|}
\hline & & & & Preparata, Tribuli Fructus \\
\hline Wu 2009 & Decoction & $30 \mathrm{~d}$, bid-tid & $\begin{array}{l}\text { 1. Xishangyihao-fang } \\
\text { 2. Xishangerhao-fang }\end{array}$ & $\begin{array}{l}\text { 1. Achyranthis Radix, Coptidis Rhizoma, Curcumae } \\
\text { Longae Radix, Scutellariae Radix, Taraxaci Herba } \\
\text { 2. Achyranthis Radix, Akebiae Caulis, Angelicae } \\
\text { Gigantis Radix, Bletillae Rhizoma, Carthami Flos, } \\
\text { Coptidis Rhizoma, Cyperi Rhizoma, Dipsaci Radix, } \\
\text { Paeoniae Radix, Persicae Semen } \\
\text { 3. Akebiae Caulis, Angelicae Gigantis Radix, Astragali } \\
\text { Radix, Chaenomelis Fructus, Cibotii Rhizoma, } \\
\text { Cistanchis Herba, Cuscutae Semen, Homalomenae } \\
\text { Rhizoma, Lumbricus, Visci Herba Et Loranthi Ramulus }\end{array}$ \\
\hline
\end{tabular}


TABle 4: Continued.

\begin{tabular}{|c|c|c|c|c|}
\hline $\begin{array}{l}\text { First } \\
\text { author }\end{array}$ & Dosage form & $\begin{array}{c}\text { Administration } \\
\text { duration and frequency }\end{array}$ & $\begin{array}{c}\text { Herbal medicine } \\
\text { name }\end{array}$ & Basic components \\
\hline $\begin{array}{l}\text { Xiong } \\
2011\end{array}$ & Decoction & $30 \mathrm{~d}$ & $\begin{array}{l}\text { 1. Xishangyihao-fang } \\
\text { 2. Xishangerhao-fang } \\
\text { 3. Xishangsanhao- } \\
\text { fang }\end{array}$ & $\begin{array}{l}\text { 1. Achyranthis Radix, Coptidis Rhizoma, Curcumae } \\
\text { Longae Radix, Scutellariae Radix, Taraxaci Herba } \\
\text { 2. achyranthis radix, akebiae caulis, angelicae gigantis } \\
\text { radix, bletillae rhizoma, carthami flos, coptidis rhizoma, } \\
\text { cyperi rhizoma, dipsaci radix, paeoniae radix, persicae } \\
\text { semen } \\
\text { 3. Akebiae Caulis, Angelicae Gigantis Radix, Astragali } \\
\text { Radix, Chaenomelis Fructus, Cibotii Rhizoma, } \\
\text { Cistanchis Herba, Cuscutae Semen, Homalomenae } \\
\text { Rhizoma, Lumbricus, Visci Herba et Loranthi Ramulus }\end{array}$ \\
\hline Xu 2017 & $\begin{array}{l}\text { Soluble granules, } \\
\text { pill preparation }\end{array}$ & More than $4 \mathrm{w}$, tid & $\begin{array}{l}\text { 1. Huoxuezhitong- } \\
\text { Jiaonang }\end{array}$ & $\begin{array}{l}\text { 1. Angelicae Gigantis Radix, Bomeolum, Eupolyphaga, } \\
\text { Notoginseng Radix, Olibanum, Pyritum } \\
\text { 2. Aconiti Lateralis Radix Preparata, Angelicae Gigantis } \\
\text { Radix, Araliae Continentalis Radix, Atractylodes } \\
\text { Macrocephala Koidzumi, Chaenomelis Fructus, } \\
\text { Cuscutae Semen, Cynomorium Songaricum Ruprecht, } \\
\text { Dipsaci Radix, Eucommiae Cortex, Gentianae } \\
\text { Macrophyllae Radix, Ginseng Radix, Lycii Fructus, } \\
\text { Osterici Radix, Paeoniae Radix, Poria Sclerotium, } \\
\text { Psoraleae Semen, Rehmanniae Radix Preparata, } \\
\text { Saposhnikoviae Radix, Testudinis Plastrum }\end{array}$ \\
\hline $\begin{array}{l}\text { Yang } \\
2018\end{array}$ & Pill preparation & $12 \mathrm{~m}, \mathrm{bid}$ & $\begin{array}{l}\text { 1. Liuwei dihuang } \\
\text { pills }\end{array}$ & $\begin{array}{l}\text { 1. Alismatis Rhizoma, Corni Fructus, Dioscoreae } \\
\text { Rhizoma, Moutan Cortex Radicis, Poria Sclerotium, } \\
\text { Rehmanniae Radix Preparata }\end{array}$ \\
\hline
\end{tabular}

Table 5: Frequently used Chinese medicinal herbs.

\begin{tabular}{|c|c|c|c|c|}
\hline Number of times used & 8 & 6 & 5 & 4 \\
\hline Chinese medicinal herbs & Angelicae Gigantis Radix & $\begin{array}{c}\text { Achyranthis Radix } \\
\text { Chaenomelis Fructus } \\
\text { Paeoniae Radix }\end{array}$ & $\begin{array}{l}\text { Cuscutae Semen } \\
\text { Persicae Semen }\end{array}$ & $\begin{array}{c}\text { Astragali Radix } \\
\text { Bletillae Rhizoma } \\
\text { Cibotii Rhizoma } \\
\text { Cistanchis Herba } \\
\text { Coptidis Rhizoma } \\
\text { Curcumae Longae Radix } \\
\text { Cyperi Rhizoma } \\
\text { Dipsaci Radix } \\
\text { Homalomenae Rhizoma } \\
\text { Rehmanniae Radix Preparata }\end{array}$ \\
\hline
\end{tabular}

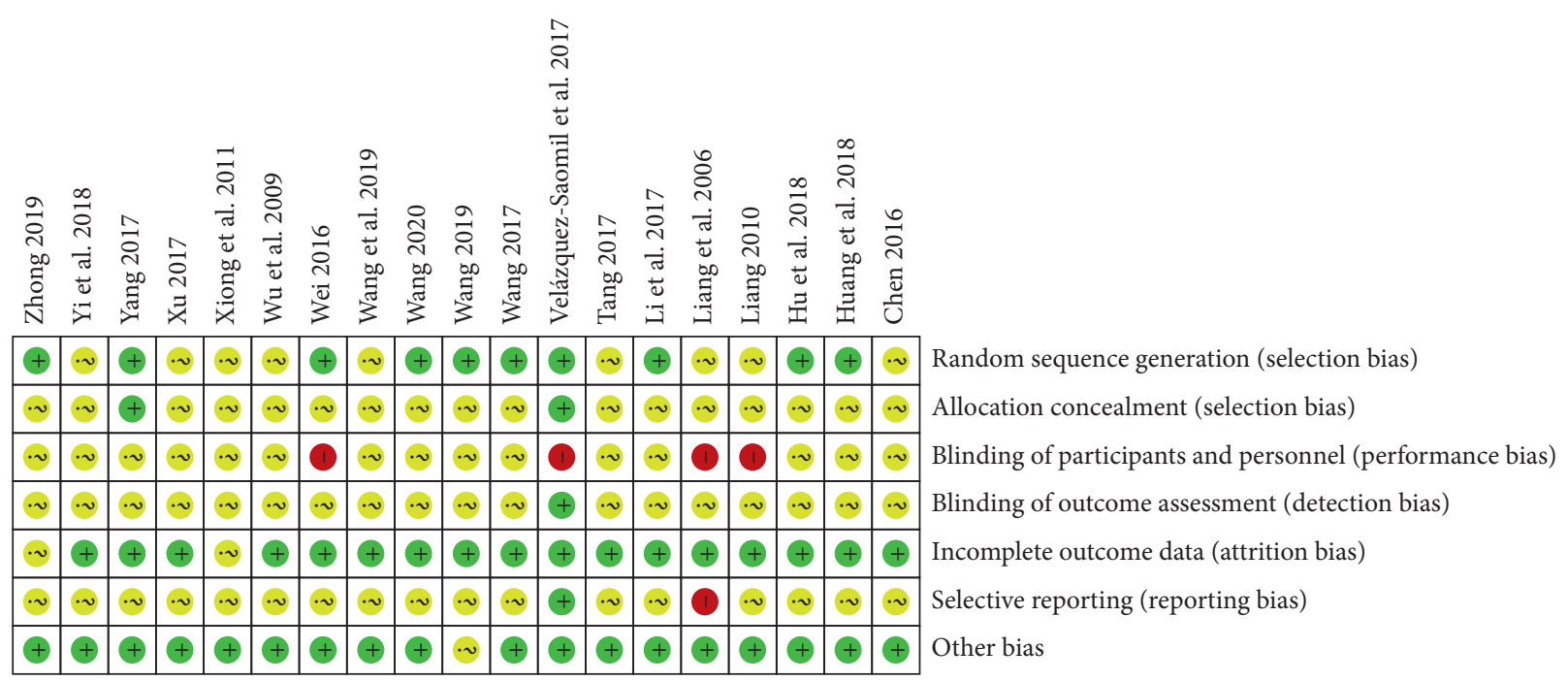

Figure 2: Assessment of the risk of bias. 


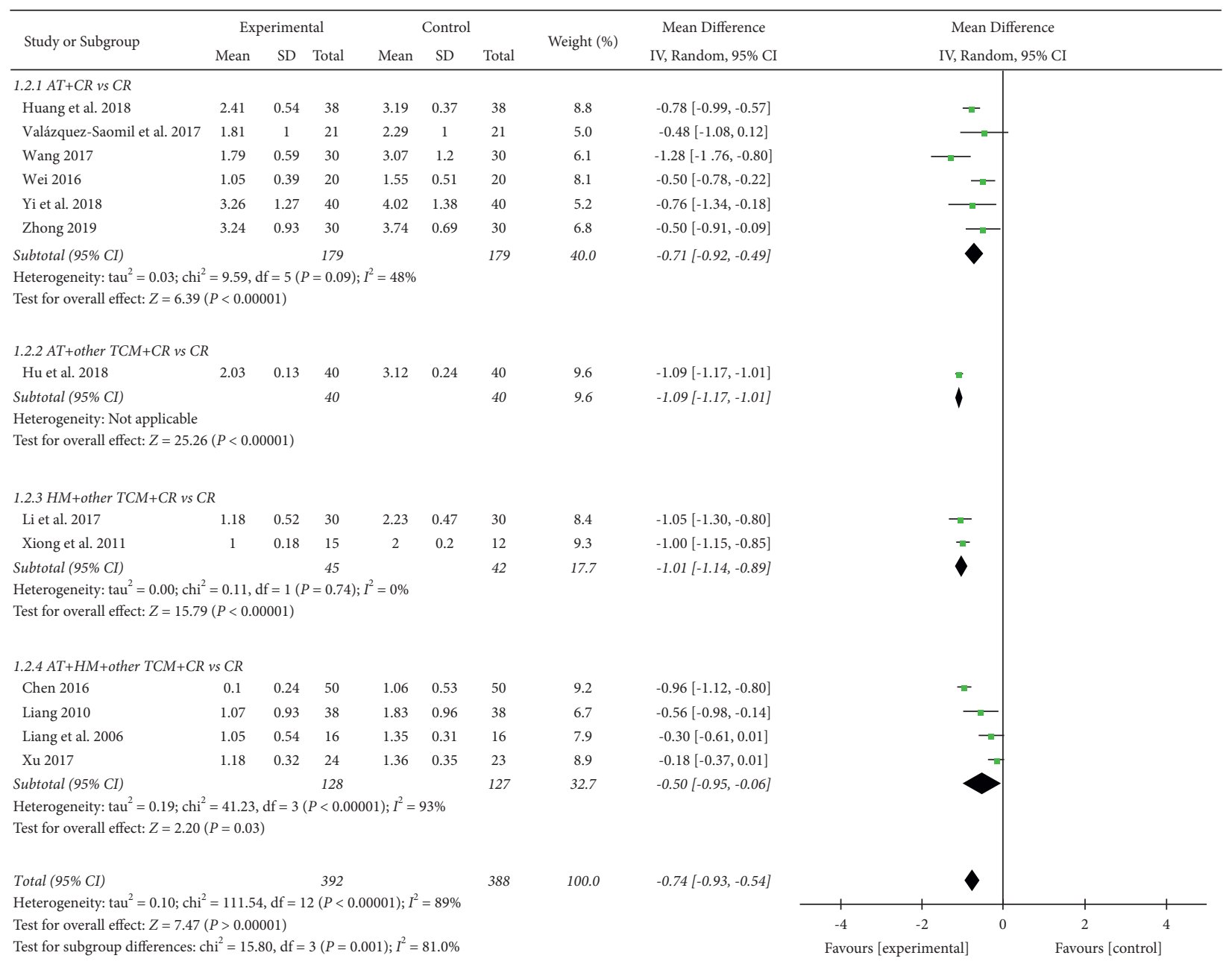

FIGURE 3: VAS score (subgroup analyzed by treatment types). AT: acupuncture treatment; CR: conventional rehabilitation; TCM: traditional Chinese medicine; HM: herbal medicine.

1.98]; $\mathrm{I} 2=0 \%$ ), and acupuncture plus herbal medicine combined with other TCM (SMD 1.17, 95\% CI [0.05, 2.29]; $\mathrm{I} 2=89 \%)$. The TCM-related improvements in ROM after ACLR were not only short-term but also long-term relative to the controls (not shown): $0-2$ weeks (SMD 1.77, 95\% CI [1.03, 2.51]), 2-4 weeks (SMD 1.82, 95\% CI [1.21, 2.43]), 4-8 weeks (SMD 1.10, 95\% CI [0.58, 1.63]; I2=62\%), $12-16$ weeks (SMD 1.54, 95\% CI [1.10, 1.99]; I2 =0\%), and more than 16 weeks (SMD 0.35, 95\% CI [0.02, 0.68]; I2=6\%).

3.4.3. Comprehensive Outcomes of the Knee Joints. Lysholm Score. In the meta-analysis of fourteen studies involving 914 subjects, the TCM group showed significantly better improvement in the Lysholm score than the control group (MD 5.62, 95\% CI [3.93, 7.32]; I2 84\%; Figure 6). All types of TCM treatments showed greater improvement in the Lysholm score except for one study using acupuncture alone: acupuncture (MD 2.74, 95\% CI [-1.31, 6.79]; $\mathrm{I} 2=69 \%$ ), herbal medicine (MD 5.08, 95\% CI [4.11, 6.05]; $\mathrm{I} 2=0 \%)$, acupuncture plus other TCM (MD 12.10, 95\% CI $[6.37,17.83]$ ), herbal medicine plus other TCM (MD 7.56,
95\% CI [2.66, 12.47]; I2 = 92\%), and acupuncture plus herbal medicine combined with other TCM (MD 5.75, 95\% CI $[2.00,9.49] ; \mathrm{I} 2=63 \%)$. Regardless of the time points of evaluation after surgery, the TCM group showed a significantly better improvement in the Lysholm score than the control group (not shown): 4-8 weeks (MD 11.75, 95\% CI $[5.85,17.64] ; \mathrm{I} 2=67 \%), 12-16$ weeks (MD 4.75, 95\% CI $[2.06,7.43] ; \mathrm{I} 2=84 \%), 16$ weeks- 1 year $(\mathrm{MD} 3.64,95 \% \mathrm{CI}$ $[2.05,5.23] ; \mathrm{I} 2=0 \%)$, and 1 year $(\mathrm{MD} 4.64,95 \% \mathrm{CI}[3.26$, $6.02]$; $\mathrm{I} 2=41 \%)$. The TCM group showed better improvement in the Lysholm score than the control group, regardless of the treatment duration (not shown): 4 weeks (MD 7.90, 95\% CI [1.33, 14.48]; I2 = 93\%), 7-8 weeks (MD 4.62, 95\% CI $[3.33,5.91] ; \mathrm{I} 2=46 \%), 12$ weeks (MD 4.64, 95\% CI [3.26, $6.02] ; \mathrm{I} 2=41 \%)$.

IKDC Subjective Score. A meta-analysis of six studies involving 376 subjects showed that there was no significant difference between the IKDC subjective scores of the TCM and control groups based on the analysis of the pooled effect (MD 3.40, 95\% CI [-0.61, 7.41]; I2 = 97\%; Figure 7). In two RCTs $[17,18]$, the TCM group showed no significant improvement in the IKDC subjective score. In the subgroup 


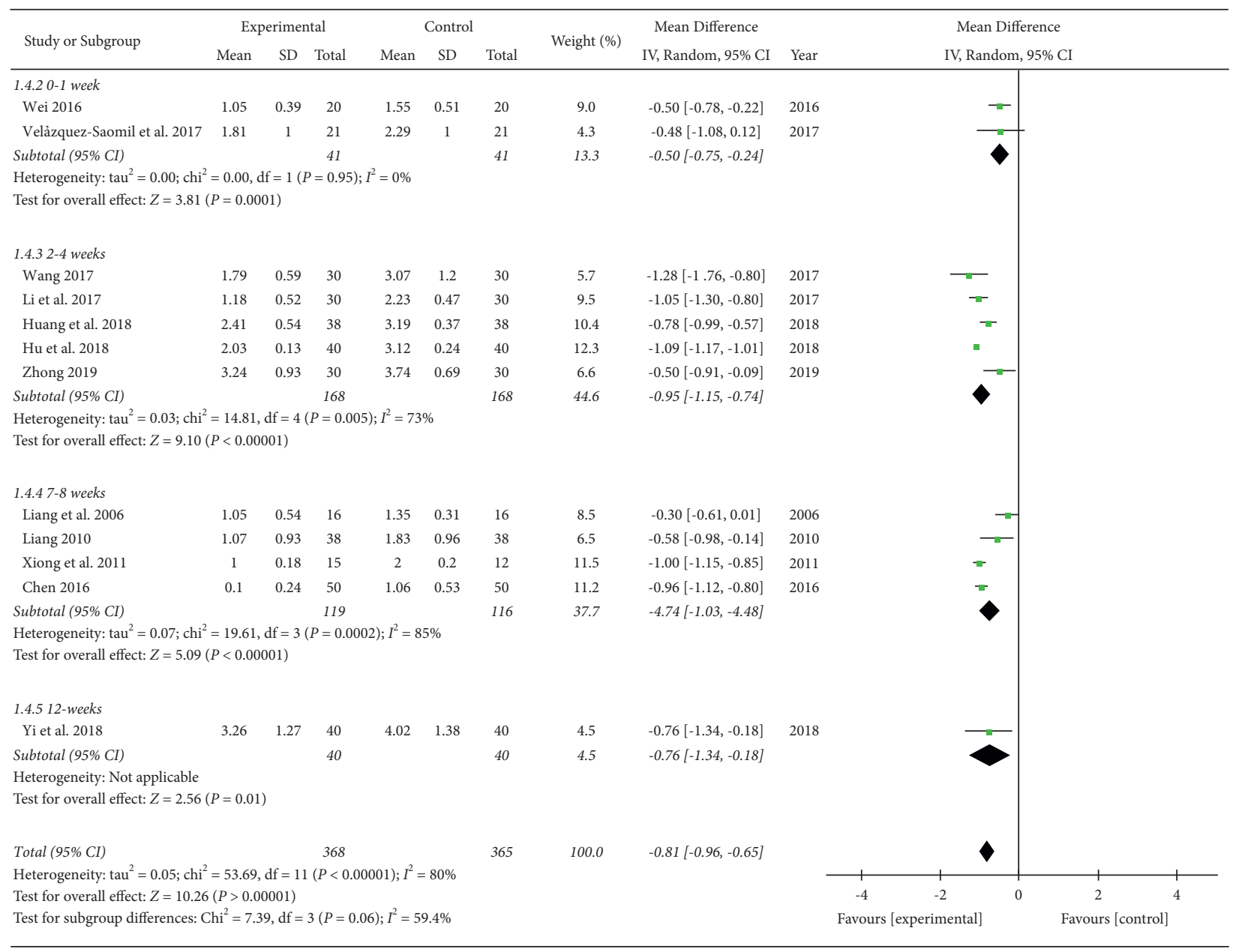

FIgURE 4: VAS score (subgroup analyzed by treatment duration).

analysis based on the TCM treatments, the herbal medicine group (MD 5.96, 95\% CI $[0.69,11.22]$; I2 = 95\%) and acupuncture plus herbal medicine combined with the other TCM group (MD 5.66, 95\% CI [2.26, 9.05]; I2 = 0\%) showed significant improvements in the IKDC subjective score, whereas the herbal medicine combined with the other TCM group showed no significant improvement in the IKDC subjective scores (MD $-0.70,95 \%$ CI $[-1.60,0.20]$; $\mathrm{I} 2=0 \%$; Figure 7). For the evaluation time points after surgery, TCM showed long-term improvement in the IKDC subjective score: $12-16$ weeks (MD $-0.51,95 \%$ CI $[-1.69,0.67$; $\mathrm{I} 2=30 \%)$, 16 weeks- 1 year $(\mathrm{MD} 6.11,95 \%$ CI $[2.02,10.20])$, and 1 year (MD 5.96, 95\% CI [0.69, 11.22]; I2 =95\%; not shown). In addition, a longer duration of treatment was associated with significant improvement in the IKDC subjective score: $7-8$ weeks (MD 1.06, 95\% CI [-1.23, 3.36]; $\mathrm{I} 2=76 \%)$ and 12 weeks (MD 5.96, 95\% CI [0.69, 11.22]; I2 = 95\%; Figure 8).

HSS Score. In the meta-analysis of three studies involving 316 subjects, the TCM group showed no significant improvement in the HSS score compared with the control group based on the pooled effect (MD 6.79, 95\% CI [-1.27, 14.86]; I2 =97\%; not shown). In the subgroup analysis, the TCM groups showed significantly better improvements in the HSS score than the control groups regardless of the treatment method (not shown): acupuncture group (MD 9.41, 95\% CI [1.62, 17.20]; I2 = 87\%) and acupuncture plus herbal medicine combined with the other TCM group (MD $2.15,95 \%$ CI $[0.38,3.92])$. The TCM groups also showed significant improvements in the HSS score compared with the control groups regardless of treatment duration (not shown): 0-2 weeks (MD 13.03, 95\% CI [10.90, 15.16]), and more than 2 weeks (MD 2.52, 95\% CI [0.63, 4.41], and $\mathrm{I} 2=6 \%)$.

Knee Circumference. In the meta-analysis of four studies involving 227 subjects, the TCM groups showed significant reductions in knee circumference compared with the control groups (SMD -1.72, 95\% CI [-2.38, -1.07]; I2 = 76\%; not shown). In the subgroup analysis based on the TCM treatments, all the subgroups showed significant reductions in the knee circumference than the control groups (not shown): acupuncture (SMD -2.65, 95\% CI $[-3.35,-1.94])$, acupuncture plus other TCM (SMD $-1.90,95 \%$ CI $[-2.43$, $-1.37]$ ), and herbal medicine plus other TCM (SMD -1.21, 95\% CI $[-1.81,-0.60] ; \mathrm{I} 2=37 \%)$. The TCM groups showed significant reductions in the knee circumference regardless of the evaluation time points after surgery (not shown): 2-4 weeks (SMD -2.65, 95\% CI $[-3.35,-1.94])$, 4-8 weeks (SMD 


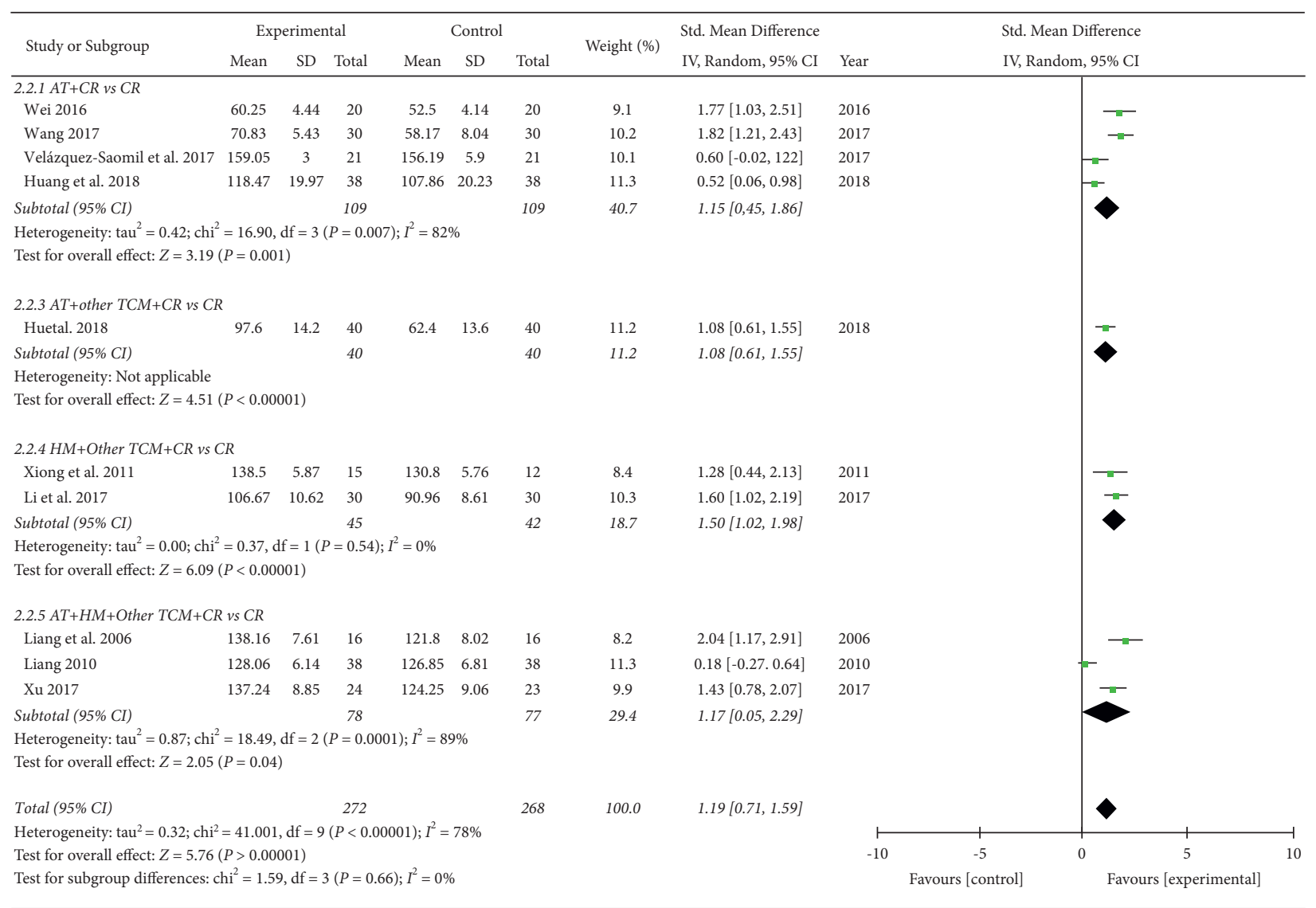

FIGURE 5: ROM (subgroup analyzed by treatment types). AT: acupuncture treatment; HM: herbal medicine; TCM: traditional Chinese medicine; CR: conventional rehabilitation.

$-1.69,95 \%$ CI $[-2.12,-1.26] ; \mathrm{I} 2=18 \%)$, and more than 8 weeks (SMD -0.83 , 95\% CI $[-1.63,-0.03])$. Treatment durations of $2-4$ weeks (SMD $-1.97,95 \%$ CI $[-2.60,-1.35]$; $\mathrm{I} 2=70 \%)$ and 7 weeks (SMD $-0.83,95 \%$ CI $[-1.63,-0.03]$ ) were also associated with significant reductions in the knee circumference (not shown).

\section{Discussion}

Appropriate postoperative care is important for satisfactory outcomes after ACLR. The medical team should always be aware of signals such as knee pain, joint stiffness, and knee oedema because planning for individual rehabilitation is based on this information [32, 33]. Current rehabilitation programs usually focus on pain control, achieving a normal ROM and reducing joint effusion [34]. A small reduction $\left(3^{\circ}-5^{\circ}\right)$ in the knee ROM after ACLR results in weaker quadriceps and increased risks of postoperative complications such as arthrofibrosis [35]. During the early postoperative phase, ambulation without pain indicates whether patients will be able to walk without crutches. During the late postoperative phase, knee joint effusion is a milestone of prognosis after ACLR [36]. The Lysholm score, IKDC subjective score, and HSS score were developed for evaluating the status of the knee joint after a ligament injury. Items for assessing the symptoms and function of the knee joint after knee surgery are included in these scales, which are commonly used for evaluating prognosis after ACLR $[37,38]$. For example, the decision to return to normal sporting activities is based on IKDC subjective scores of more than 70 points [12]. Therefore, we selected the VAS score as the primary outcome, while ROM, knee circumference, Lysholm score, IKDC subjective score, and HSS score were chosen as the secondary outcomes in this review.

Currently, there are several rehabilitation protocols for postoperative care after ACLR. However, the most appropriate rehabilitation modality is still being debated on $[2,3]$. A consensus cannot be reached because the postoperative conditions of patients differ in each case. Postoperative conditions are affected by various symptoms and the function of the knee joint, which may interrupt scheduled rehabilitation programs. Therefore, there is an increasing demand for updated rehabilitation programs for relieving symptoms and promoting the function of the knee joint after ACLR [4].

For these reasons, TCM may be used as adjuvant therapy for rehabilitation after ACLR because it is a more active intervention in that it can directly stimulate the muscles around the knee joint and promote systemic recovery compared to conventional rehabilitation. A recent systematic review revealed that the combination of TCM and CPM can promote better recovery of the knee-joint function after knee surgeries, including ACLR, TKA, and OR/IF versus 


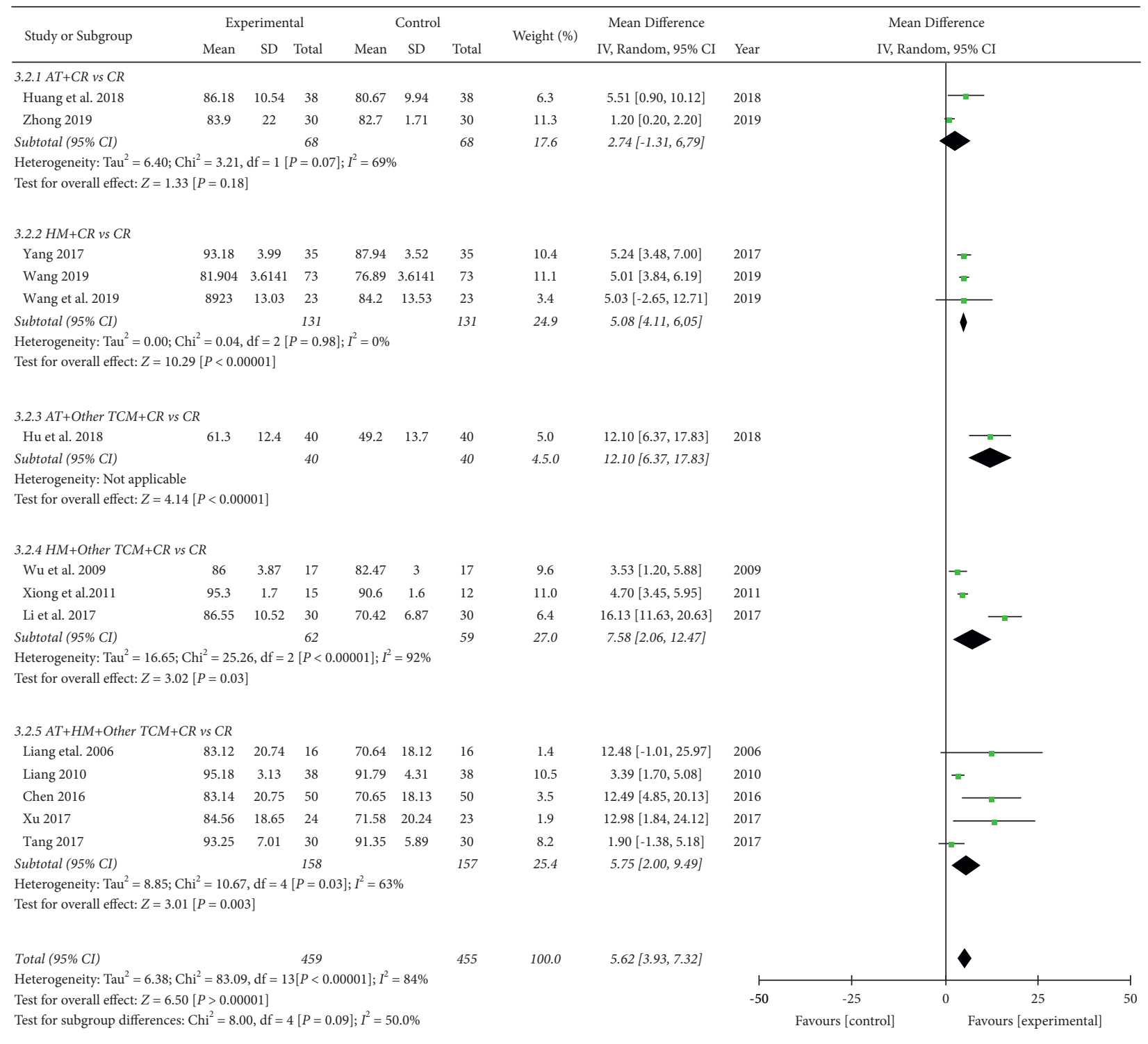

Figure 6: Lysholm score (subgroup analyzed by treatment types). AT: acupuncture treatment; HM: herbal medicine; TCM: traditional Chinese medicine; CR: conventional rehabilitation.

CPM alone [39]. Therefore, we conducted a systematic review and meta-analysis to verify the clinical effects of TCM combined with conventional rehabilitation during postoperative care after ACLR.

This meta-analysis found that TCM combined with conventional rehabilitation can improve postoperative pain after ACLR. The VAS score, which was the primary outcome of this review, significantly decreased in the TCM groups regardless of the TCM treatment type, evaluation time points, and treatment duration. The knee ROM also significantly increased regardless of TCM intervention or evaluation time points after surgery. This meta-analysis also revealed that TCM treatments can significantly reduce knee swelling after ACLR regardless of their types, evaluation time points, and treatment duration. These results may be attributed to the analgesic effect of acupuncture treatment and the anti-inflammatory effect of herbal medicine.
The most frequently used acupuncture point was SP10, which is located in the belly of the vastus medialis muscle. Moreover, 10 most frequently used acupuncture points were all located within the affected lower limb, especially at the quadriceps femoris, tibialis anterior, and the gastrocnemius muscle. Unlike this review, a recent systematic review [6] and retrospective study [40] dealt mostly with trials in which acupuncture treatments were applied on the distal or contralateral part of the operated limb after TKA. While those studies validated the analgesic effect of acupuncture that lasted for a short-term only, this metaanalysis proved that the analgesic effect of localized acupuncture treatment in affected knees lasted for a up to one year. According to an RCT comparing the effect of using local acupoints and distal acupoints in degenerative knee osteoarthritis, localized acupuncture was more effective in improving the Western Ontario and McMaster 


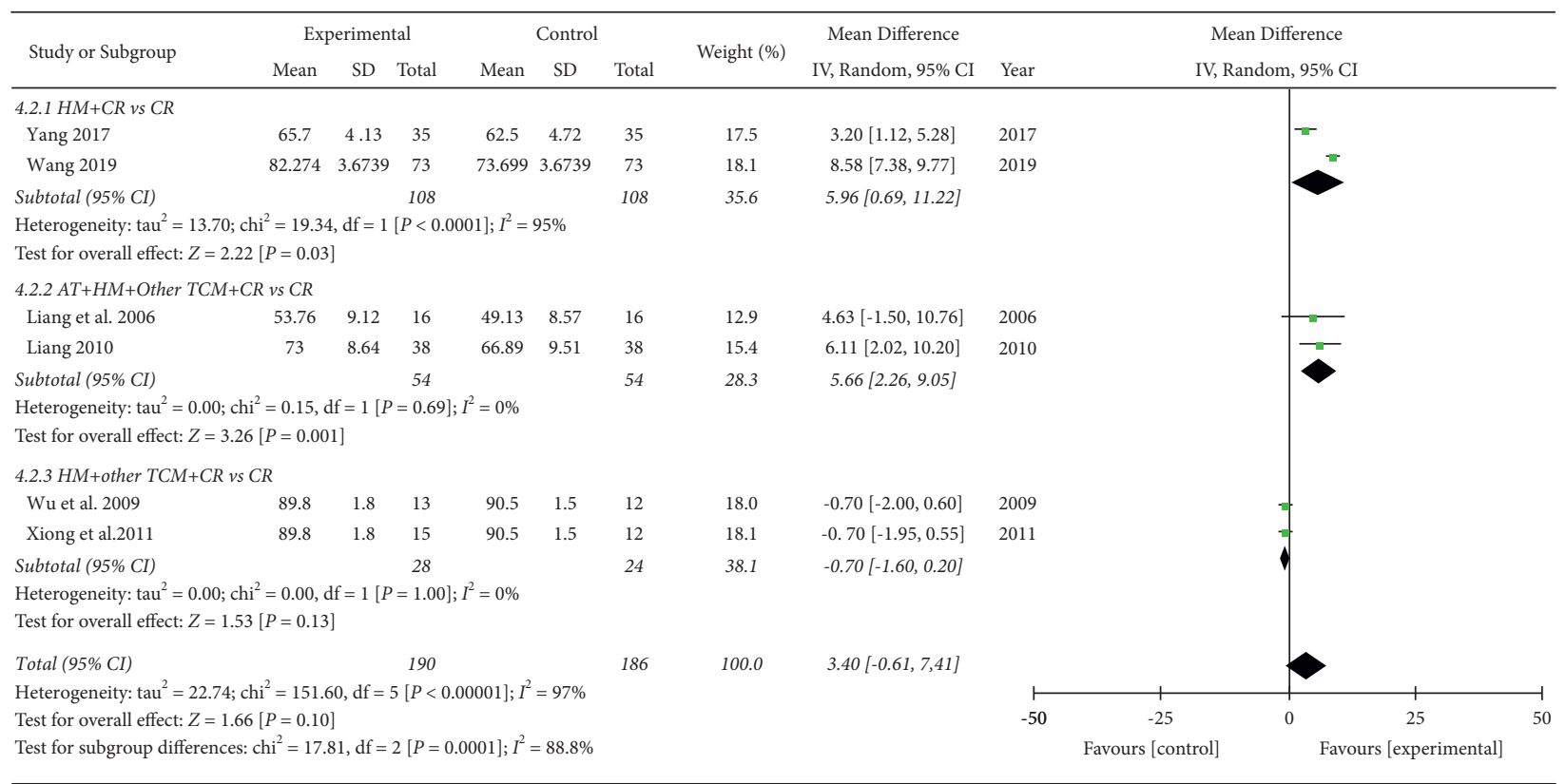

FIGURE 7: IKDC subjective score (subgroup analyzed by treatment types). AT: acupuncture treatment; HM: herbal medicine; TCM: traditional Chinese medicine; CR: conventional rehabilitation.

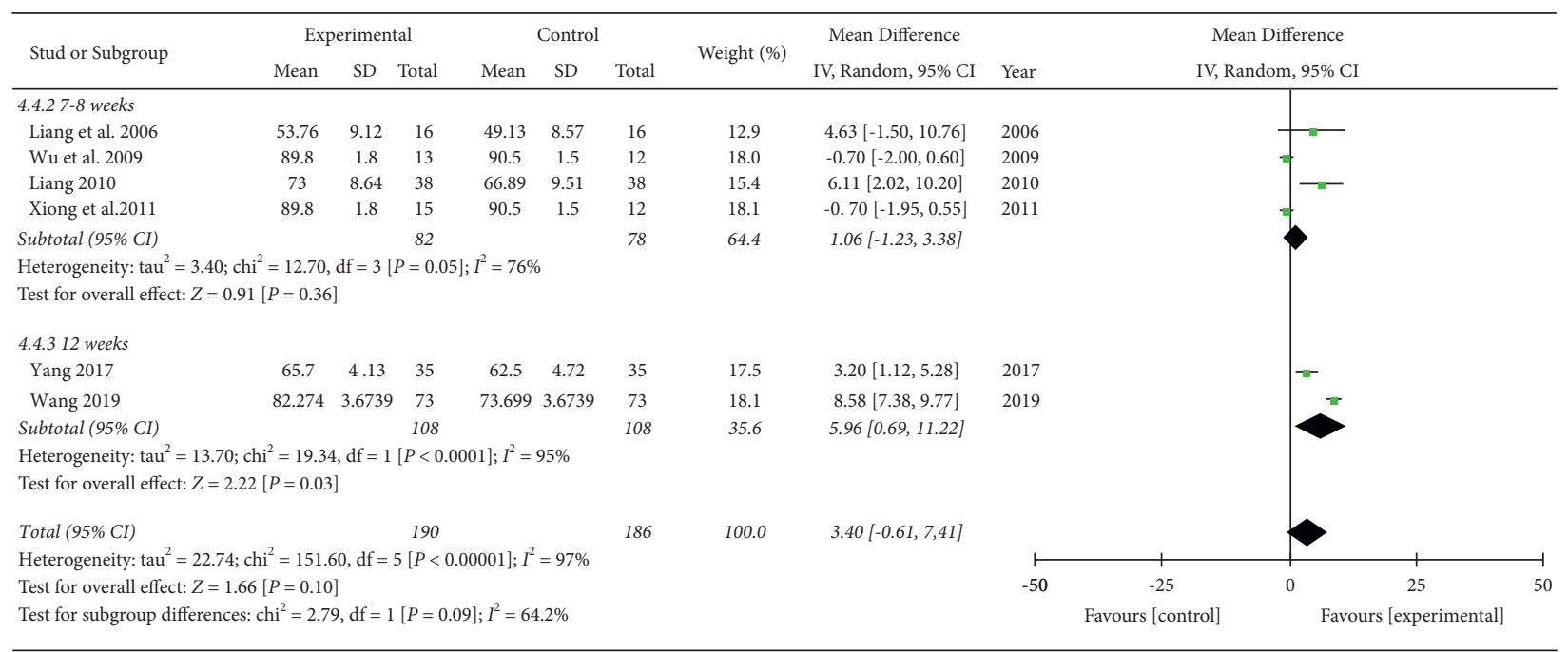

FIGURE 8: IKDC subjective score (subgroup analyzed by treatment duration).

Universities Osteoarthritis Index (WOMAC) score by directly stimulating the structures around the knee joint [41]. The localized analgesic effect of acupuncture can be achieved through adenosine $\mathrm{A} 1$ receptor mediation and axonal reflex, which stimulate the secretion of neuropeptides such as calcitonin gene-related peptide (CGRP), adenosine, and nitric oxide (NO). These substances dilate blood vessels and promote blood circulation [42, 43]. Acupuncture also has a segmental effect mediated by the gate control theory in addition to its regulation of the descending inhibitory system at the subcortical and cortical levels [44]. Moreover, the restriction of ROM after surgery is often caused by the stiffness of muscles around the knee joint $[34,45]$. Therefore, acupuncture treatment at these muscles may improve the ROM of the knee joint by promoting blood flow around the knee joint [46].

Herbal medicine is often used for knee osteoarthritis and its analgesic effect is proven in several systematic reviews $[47,48]$. Herbal medicine can also reduce the incidence rates of deep vein thrombosis after lower extremity orthopedic surgery [49]. Angelicae Gigantis Radix, which was used most frequently in the RCTs, is known to demonstrate an anti-inflammatory effect by inhibiting proinflammatory mediators such as the tumor necrosis factoralpha (TNF- $\alpha$ ), interleukin-6 (IL-6), IL-10, and vascular endothelial growth factor (VEGF) in lipopolysaccharide (LPS)-stimulated mouse macrophages [50]. The second most used components in herbal medicine were Paeoniae 
Radix, Achyranthis Radix, and Chaenomelis Fructus. Monoterpenoids in Paeoniae Radix serve as potential leads for the development of anti-inflammatory agents [51]. Achyranthis Radix usually serves as a lower-guiding drug and also enhances the therapeutic effect of TCM on the lower limbs and improves the supply of blood in the inflamed joint [52]. Moreover, quercetin in Chaenomelis Fructus can be a potent source for anti-inflammatory agents [53], and recent studies have shown that its components have anti-inflammatory properties which were effective for arthritis in a rat model [54].

Among the scales used for the comprehensive evaluation of the knee joint after ACLR, only the Lysholm score significantly improved in the TCM group based on the analysis of the pooled effect. Owing to the considerable heterogeneity involved, we conducted a subgroup analysis. During the subgroup analysis of Lysholm scores and IKDC subjective scores based on the TCM treatment types, the acupuncture groups and the herbal medicine plus other TCM treatment groups showed no improvement in Lysholm scores and IKDC subjective scores, respectively. However, the TCM groups, including both acupuncture and herbal medicine, showed significant improvements in Lysholm scores and IKDC subjective scores with low heterogeneity, which suggests that more diverse TCM treatments tend to be effective for relieving symptoms and promoting the function of the knee joint after ACLR. In the subgroup analysis of the IKDC subjective score based on the evaluation time points, TCM had begun to show effects in the past 16 weeks at the earliest with a relatively long treatment duration of 12 weeks. A report of a clinically important difference in the IKDC subjective score after 11.5-20.5 (range 6-28) months in patients who underwent surgical procedures of the knee joint may explain this [55]. In the subgroup analysis of the HSS score based on the treatment duration, TCM was associated with significant improvements in all the subgroups with low heterogeneity.

Three trials reported adverse events, including hemorrhage or dizziness after acupuncture and diarrhea after taking herbal medicine. However, these cases were mild, and the symptoms resolved spontaneously without lifethreatening complications. In one study [16], acupuncture was associated with significantly fewer adverse events than taking analgesics. Some systematic reviews reported cases of local infection after acupuncture treatment [56, 57]. However, in this review, although acupuncture points used in the included RCTs were usually near the affected knee joint, serious adverse events such as surgical site infection did not occur. Therefore, this systematic review showed that TCM treatments for patients after ACLR were comparatively safe. However, further studies are needed to validate our findings because only a few of the included RCTs reported adverse events.

All the included studies were RCTs. Among the seven domains in ROB, performance bias was mainly assessed because it can affect subjective outcomes such as the VAS score, Lysholm score, IKDC subjective score, and HSS score [58]. The experimental groups were treated with TCM treatments and conventional rehabilitation, whereas the control groups were treated with conventional rehabilitation alone; therefore, it seemed impossible to blind the participants completely from the TCM treatments. Accordingly, performance bias was considered not clear or relatively high in all the included studies, and the quality of evidence was downgraded by one level for the ROB of the subjective outcomes. The quality of evidence was also downgraded by one for the inconsistency of the results because of the considerable heterogeneity although it changed during the subgroup analysis. There was no evidence of indirectness because all the studies directly compared the interventions. Most studies used adequate population sizes. However, in relation to the IKDC subjective score, HSS score, and knee circumference, the population size was relatively low, and the quality of evidence was downgraded by one for each outcome. The level of evidence and the reasons for the upgrades and downgrades are shown in Table 6 . The ratings for the quality of evidence for the overall outcomes ranged from moderate to very low because of performance bias and obvious heterogeneities. The ratings for evidence quality were as follows: moderate for ROM; low for the VAS, Lysholm scores, and knee circumferences; and very low for the IKDC subjective and HSS scores.

There are several limitations to this review. First, owing to the inconsistency of the TCM intervention types, duration, and evaluation time points, high heterogeneity was observed during the meta-analysis, although part of it was offset by the subgroup analysis. For higher quality metaanalyses, RCTs that adopted standardized TCM treatments for ACLR patients should be published in the future. Second, several studies with high risks of bias were included in this review. Well-designed RCTs with high-quality methodologies should be conducted in the future. Third, most of the included studies were conducted in China [13-30] except for one study from Spain [31]. Since TCM treatments are commonly performed in China, their cultural customs may act as another source of bias in this review. Therefore, more geographically diverse RCTs are encouraged in the future.

In summary, TCM can relieve pain, enhance the mobility of the knee joint, reduce edema after surgery, and improve several symptoms and the function of the knee joint after ACLR. In addition, the TCM treatments that were more diverse and had longer treatment durations and evaluation time points were more effective at improving the Lysholm scores and IKDC subjective scores after ACLR. Despite these promising results, the strength of evidence is weak and no definite conclusions can be drawn, given that the overall methodological quality of the studies was relatively low and the heterogeneity was not entirely resolved by the subgroup analysis. Nevertheless, this paper is the first systematic review to evaluate the efficacy of postoperative care using TCM after ACLR and will be a useful cornerstone for future research on TCM for postoperative care after various musculoskeletal surgeries. 
TABLE 6: Summary of findings.

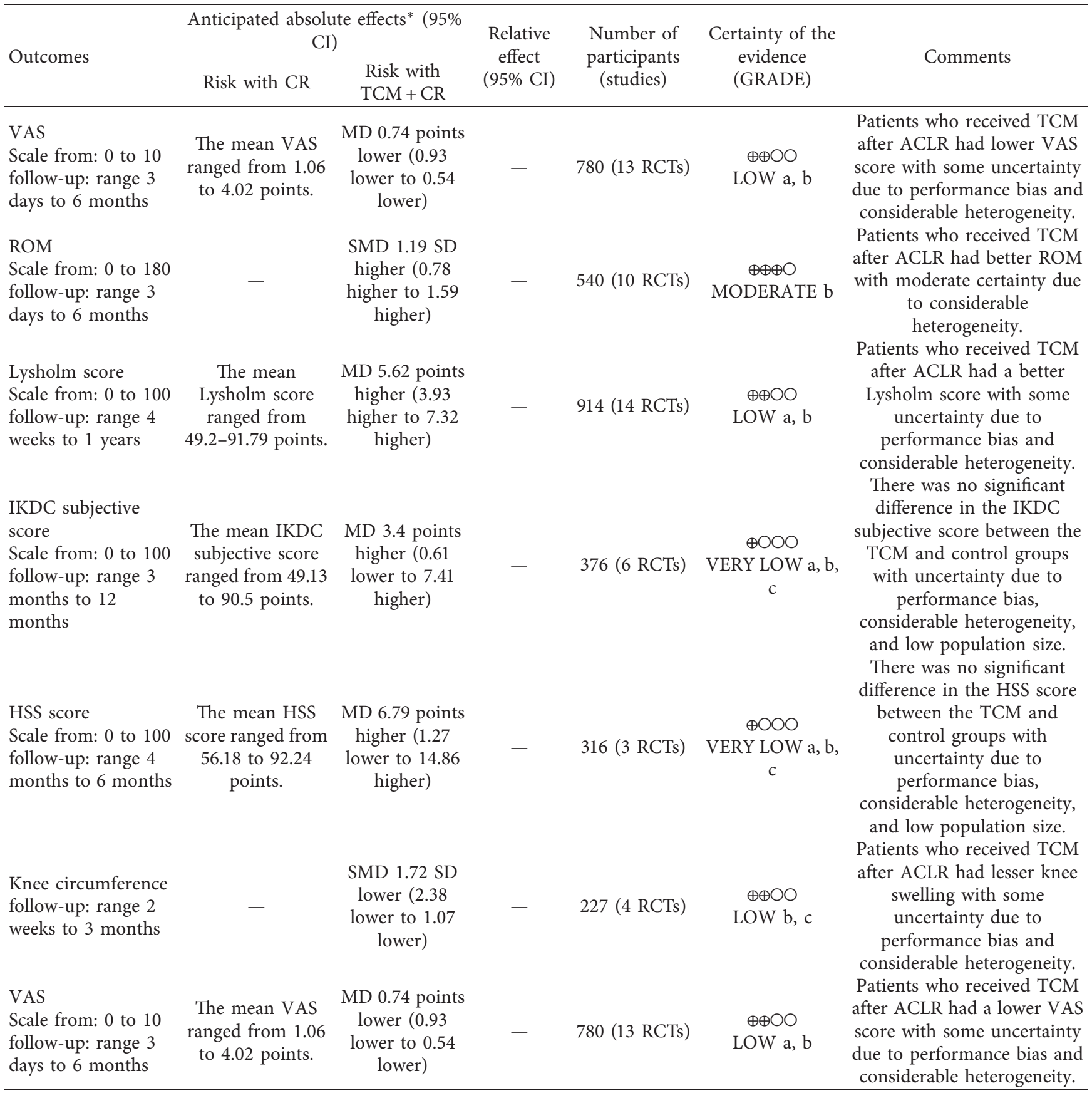

${ }^{\mathrm{a}}$ Unclear performance bias which may affect outcomes. ${ }^{\mathrm{b}}$ Considerable heterogeneity was detected. However, most inconsistency was explained by differences in interventions, duration, and evaluation time points. ${ }^{c}$ Population size less than 400. CI: confidence interval; MD: mean difference; SMD: standardized mean difference; VAS: visual analogue scale; ROM: range of motion; IKDC: International knee documentation committee; HSS: hospital for special surgery; RCTs: randomized controlled trials; ACLR: anterior cruciate ligament reconstruction; TCM: traditional Chinese medicine.

\section{Conclusions}

TCM can be combined with conventional rehabilitation to reduce postoperative pain and knee edema as well as increase the mobility and function of the knee joint after ACLR. However, this recommendation should be carefully applied in clinical practice because of the relatively low overall quality of the included RCTs. RCTs with high methodological quality and more standardized TCM treatments should be conducted in the future.

\section{Abbreviations}

ACL: Anterior cruciate ligament

ACLR: Anterior cruciate ligament reconstruction

CGRP: Calcitonin gene-related peptide 


$\begin{array}{ll}\text { CI: } & \text { Confidence intervals } \\ \text { CNKI: } & \text { Chinese National Knowledge Infrastructure } \\ \text { CPM: } & \text { Continuous passive motion therapy } \\ \text { CR: } & \text { Conventional rehabilitation } \\ \text { EA: } & \text { Electroacupuncture } \\ \text { HSS: } & \text { Hospital for special surgery } \\ \text { IKDC: } & \text { International Knee Documentation Committee } \\ & \text { 2000 } \\ \text { MDs: } & \text { Mean differences } \\ \text { NO: } & \text { Nitric oxide } \\ \text { NMES: } & \text { Neuromuscular electrical stimulation } \\ \text { OR/IF: } & \text { Open reduction and internal fixation } \\ \text { RCT: } & \text { Randomized controlled trial } \\ \text { ROM: } & \text { Range of motion } \\ \text { SMDs: } & \text { Standardized mean differences } \\ \text { TCM: } & \text { Traditional Chinese medicine } \\ \text { TKA: } & \text { Total knee arthroplasty } \\ \text { TKR: } & \text { Total knee replacement } \\ \text { TrPs: } & \text { Trigger points } \\ \text { VAS: } & \text { Visual analogue scale } \\ \text { WMDs: } & \text { Weighted mean differences } \\ \text { WOMAC: } & \text { Western Ontario and Mcmaster Universities } \\ & \text { Osteoarthritis Index } \\ \text { JMAS: } & \text { Japan Medical Abstracts Society. } \\ & \end{array}$

\section{Appendix}

Search strategies for MEDLINE/PubMed databases are as follows (Access Date: 7 June 2020):
(1) anterior cruciate ligament reconstruction [MeSH]
(2) anterior cruciate ligament injuries [MeSH]
(3) “anterior cruciate ligament repair" [tw]
(4) “anterior cruciate ligament" [tw]
(5) 1 OR 2 OR 3 OR 4
(6) “anterior cruciate ligament" [tw]
(7) “intra-articular knee ligament" [tw]
(8) 6 OR 7
(9) injury OR rupture OR torn OR destruction OR trauma OR reconstruction OR repair

(10) 8 AND 9

(11) 5 OR 10

(12) acupuncture therapy [MeSH]

(13) acupuncture [MeSH]

(14) acupuncture point [MeSH]

(15) “acupuncture needle" [tw]

(16) meridians [MeSH]

(17) acupuncture* [tw]

(18) needle* [tw]

(19) acupoint* [tw]

(20) electroacupuncture [MeSH]

(21) electroacupuncture [tw]

(22) pharmacoacupunctur* [tw]
(23) pharmacoacupunctur* [tw]

(24) "acupoint injection" [tw]

(25) "auricular acupunctur*" [tw]

(26) “ear acupunctur*” [tw]

(27) “auricular needl*” [tw]

(28) “ear needl*" [tw]

(29) “fire acupunctur*" [tw])

(32) "scalp acupuncture*" [tw]

(30) “warm acupunctur*” [tw]

(31) “warm needl*” [tw]

(33) 12 OR 13 OR 14 OR 15 OR 16 OR 17 OR 18 OR 19 OR 20 OR 21 OR 22 OR 23 OR 24 OR 25 OR 26 OR 27 OR 28 OR 29 OR 30 OR 31 OR 32

(34) Medicine, Chinese Traditional [MeSH]

(35) Chinese herbal medicine [tw]

(36) Chinese medicine [tw]

(37) Chinese herbal drug [tw]

(38) traditional herbal medicine [tw]

(39) herbal medicine [tw]

(40) decoction [tw]

(41) tang $[\mathrm{tw}]$

(42) *tang [tw]

(43) formula $[\mathrm{tw}]$

(44) 34 OR 35 OR 36 OR 37 OR 38 OR 39 OR 40 OR 41 OR 42 OR 43

(45) 33 OR 44

(46) "randomized controlled trial" [Publication Type]

(47) "randomized controlled trials as topic" [MeSH]

(48) "random allocation" [MeSH]

(49) "double-blind method" [MeSH]

(50) "single-blind method" [MeSH]

(51) placebo $[\mathrm{MeSH}]$

(52) random* $^{*}[\mathrm{tw}]$

(53) $\mathrm{rct}[\mathrm{tw}]$

(54) rct's $[\mathrm{tw}]$

(55) rcts [tw]

(56) placebo* $\left.^{*} \mathrm{tw}\right]$

(57) 46 OR 47 OR 48 OR 49 OR 50 OR 51 OR 52 OR 53 OR 54 OR 55 OR 56

(58) 11 AND 45 AND 57 Search Results: 14

Search strategies for EMBASE database are as follows (Access Date: 7 June 2020):

(31) (13) AND (20) AND (30)

(30) (21) OR (22) OR (23) OR (24) OR (25) OR (26) OR (27) OR (28) OR (29)

(29) placebo*: ab, ti 
(28) rcts: ab, ti

(27) rct: ab, ti

(26) random* $^{*} \mathrm{ab}$, ti

(25) “placebo"/exp

(24) "single blind procedure"/exp

(23) "double blind procedure"/exp

(22) "randomization"/exp

(21) "randomized controlled trial (topic)"/exp

(20) (14) OR (15) OR (16) OR (17) OR (18) OR (19)

(19) “formula”:ab, ti

(18) “-tang":ab, ti

(17) “decoction":ab, ti

(16) "herbal medicine"/exp

(15) “Chinese medicine equipment"/exp

(14) "Chinese medicine"/exp

(13) (5) OR (12)

(12) (8) AND (11)

(11) (9) OR (10)

(10) reconstruction:ab, ti OR repair:ab, ti

(9) injury:ab, ti OR rupture:ab, ti OR torn:ab, ti OR destruction:ab, ti OR trauma:ab, ti

(8) (6) OR (7)

(7) "intra-articular knee ligament": ab, ti

(6) "anterior cruciate ligament": ab, ti

(5) (1) OR (2) OR (3) OR (4)

(4) "anterior cruciate ligament": ab, ti

(3) "anterior cruciate ligament repair": ab, ti

(2) “anterior cruciate ligament injury"/exp

(1) "anterior cruciate ligament reconstruction"/exp Search Results: 21

Search strategies for CENTRAL database are as follows (Access Date: 7 June 2020):

(1) $\mathrm{MeSH}$ descriptor: [Anterior Cruciate Ligament Reconstruction] explode all trees

(2) $\mathrm{MeSH}$ descriptor: [Anterior Cruciate Ligament Injuries] explode all trees

(3) "Anterior Cruciate Ligament repair"

(4) "Anterior Cruciate Ligament"

(5) (1) OR (2) OR (3) OR (4)

(6) "anterior cruciate ligament"

(7) "intraarticular knee ligament"

(8) (6) OR (7)

(9) (injury OR rupture OR torn OR destruction OR trauma)

(10) (reconstructions OR repair)

(11) (9) OR (10)

(12) (8) AND (11)
(13) (5) OR (12)

(14) MeSH descriptor: [Medicine, Chinese Traditional] explode all trees

(15) $\mathrm{MeSH}$ descriptor: [Herbal Medicine] explode all trees

(16) MeSH descriptor: [Drugs, Chinese Herbal] explode all trees

(17) traditional herbal medicine

(18) decoction

(19) formula

(20) * tang

(21) (14) OR (15) OR (16) OR (17) OR (18) OR (19) OR (20)

(22) $\mathrm{MeSH}$ descriptor: [Randomized Controlled Trial] explode all trees

(23) MeSH descriptor: [Randomized Controlled Trials as Topic] explode all trees

(24) MeSH descriptor: [Random Allocation] explode all trees

(25) $\mathrm{MeSH}$ descriptor: [Double-Blind Method] explode all trees

(26) $\mathrm{MeSH}$ descriptor: [Single-Blind Method] explode all trees

(27) MeSH descriptor: [Placebos] explode all trees

(28) random*

(29) $\mathrm{rct}$

(30) rct's

(31) rcts

(32) placebo*

(33) (22) OR (23) OR (24) OR (25) OR (26) OR (27) OR (28) OR (29) OR (30) OR (31) OR (32)

(34) (13) AND (21) AND (33)

Search Results: 12

Search strategies for CNKI database are as follows (Access Date: 7 June 2020):

Subject category: Medicine and Public Health

Sub-database: Journal articles, Dissertations

Search strategy:

(SU = “前交叉韧带”+“前交叉韧带重建术”) AND (SU = “中医治疗” + “中医治疗结合” + “针炎” + “针 刺”+ “中药”)

Search Results: 207

\section{Data Availability}

Data will be provided upon request to the corresponding author.

\section{Disclosure}

H. C. and H. K. are the co-first authors. 


\section{Conflicts of Interest}

The authors declare no conflicts of interest.

\section{Authors' Contributions}

H. C. and H. K. designed the main concept of the study and participated in data acquisition, data analysis, data interpretation, manuscript drafting, revision, and final reviewing of the manuscript. W. C., M. S., J. C., and K. K. revised the manuscript and supervised the research. H. C. and H. K. are contributed equally to this study.

\section{Acknowledgments}

This research was supported by a grant of the Korea Health Technology R\&D Project through the Korea Health Industry Development Institute (KHIDI), funded by the Ministry of Health and Welfare, Republic of Korea (grant number: HI20C1405). The corresponding author Won-Seok Chung received the grant with responsibility.

\section{References}

[1] T. L. Sanders, H. Maradit Kremers, A. J. Bryan et al., "Incidence of anterior cruciate ligament tears and reconstruction," The American Journal of Sports Medicine, vol. 44, no. 6, pp. 1502-1507, 2016.

[2] N. Van Melick, R. E. H. Van Cingel, F. Brooijmans et al., "Evidence-based clinical practice update: practice guidelines for anterior cruciate ligament rehabilitation based on a systematic review and multidisciplinary consensus," British Journal of Sports Medicine, vol. 50, no. 24, pp. 1506-1515, 2016.

[3] L. M. Kruse, B. Gray, and R. W. Wright, "Rehabilitation after anterior cruciate ligament reconstruction," Journal of Bone and Joint Surgery, vol. 94, no. 19, pp. 1737-1748, 2012.

[4] M.-Y. Song, H.-G. Jo, J.-U. Sul, and J. Leem, "Proposal of eastwest integrative medicine manual for rehabilitation after knee surgery," Journal of Korean Medicine Rehabilitation, vol. 28, no. 1, pp. 97-107, 2018.

[5] K. Zippelius, "The integrative TCM treatment of cruciate ligament injury," Journal of Chinese Medicine, vol. 114, pp. 5-15, 2017.

[6] D. Tedesco, D. Gori, K. R. Desai et al., "Drug-free interventions to reduce pain or opioid consumption after total knee arthroplasty," JAMA Surgery, vol. 152, no. 10, pp. e172872-13, 2017.

[7] C. Yue, X. Zhang, Y. Zhu, Y. Jia, H. Wang, and Y. Liu, "Systematic review of three electrical stimulation techniques for rehabilitation after total knee arthroplasty," The Journal of Arthroplasty, vol. 33, no. 7, pp. 2330-2337, 2018.

[8] T. Barlow, C. Downham, and D. Barlow, "The effect of complementary therapies on post-operative pain control in ambulatory knee surgery: a systematic review," Complementary Therapies in Medicine, vol. 21, no. 5, pp. 529-534, 2013.

[9] M. J. Page, J. E. McKenzie, P. M. Bossuyt et al., “The PRISMA 2020 statement: an updated guideline for reporting systematic reviews," BMJ, vol. 372, p. n71, 2021.

[10] H. Kim and W.-S. Chung, "Acupuncture and herbal medicine for postoperative care following anterior cruciate ligament reconstruction," Medicine, vol. 100, no. 8, pp. e24936-e24944, 2021.

[11] J. P. T. Higgins, S. G. Thompson, J. J. Deeks, and D. G. Altman, "Measuring inconsistency in meta-analyses," BMJ, vol. 327, no. 7414, pp. 557-560, 2003.

[12] S. B. Brotzman and C. Manske R, Clinical Orthopaedic Rehabilitation: An Evidence-Based Approach, Hanmibook, Seoul, Republic of Korea, 3rd edition, 2012.

[13] F. Chen, "Effect of traditional Chinese medicine treatment combined with rehabilitation training on early knee joint function after arthroscopic anterior cruciate ligament reconstruction," journal Theory and Practice in Medicine, vol. 29, no. 2, pp. 207-209, 2016.

[14] K. P. Hu, Y. Q. Tan, and Y. M. Chen, "Study on the early application of Traditional Chinese Medicine after Anterior cruciate ligament reconstruction," Journal of Emergency in Traditional Chinese Medicine, vol. 27, no. 4, pp. 750-752, 2018.

[15] W. X. Wang, Clinical Observation on 60 Cases of Acupuncture and Moxibustion Combined with Rehabilitation after Anterior Cruciate Ligament ReconstructionLiaoning University of Traditional Chinese Medicine, Shenyang, China, 2017, https:// oversea.cnki.net/kns/detail/detail.aspx? FileName $=1017865302$. nh\&DbName $=$ CMFD2018.

[16] C. Wei, The Study of the Preemptive Analgesia Effect of Acupuncture at Postoperative Pain after Anterior Cruciate Ligament ReconstructionGuangzhou University of Chinese, Guangzhou, China, 2016, https://oversea.cnki.net/kns/detail/ detail.aspx?FileName=1016208532. nh\&DbName $=$ CMFD2017.

[17] J. Wu, S. H. Xie, S. Y. Li, and X. H. Cheng, "13 cases of early rehabilitation treatment with integrated traditional Chinese medicine and western medicine after anterior cruciate ligament reconstruction with human allograft under arthroscopy," Jiangxi University of Traditional Chinese Medicine, vol. 25, no. 4, pp. 45-46, 2009.

[18] Q. Xiong and Y. H. Chen, "Efficacy observation of different methods of rehabilitation care after anterior cruciate ligament reconstruction," MOD Diagnosis Treatment, vol. 22, no. 3, pp. 148-149, 2011.

[19] D. Xu, X. H. Wang, S. J. Wei, L. L. Zhao, F. Zhang, and J. Liu, "Treating 24 cases of knee anterior cruciate ligament reconstruction by integrative medicine rehabilitation therapy," Journal of Traditional Chinese Medical Sciences, vol. 30, no. 8, pp. 117-119, 2017.

[20] W. Y. Yang, J. K. Pan, H. Xie, Z. M. Zhang, X. W. Cao, and J. Liu, "Effect of kidney-tonifying recipe on tendon-bone healing after anterior cruciate ligament reconstruction," Chinese Journal of Tissue Engineering, vol. 21, no. 4, pp. 591-597, 2017.

[21] X. Q. Yi and Q. Tiang, "Study on the effect of joint loosening technique combined with acupuncture on walking ability after anterior cruciate ligament reconstruction," China Foreign Medical Treatment.vol. 37, no. 20, pp. 154-156, 2018.

[22] X. W. Zhong, Clinical Observation of Quadriceps Femoris Atrophy after Anterior Cruciate Ligament Reconstruction by AcupunctureFujian University of Traditional Chinese Medicine, Fuzhou, China, 2019, https://oversea.cnki.net/kns/ detail/detail.aspx?FileName $=1019866700$. nh\&DbName $=$ CMFD2019.

[23] C. R. Huang, C. Y. Zhao, B. B. Zhu, and Y. Y. Xiao, "Effect of warming needle moxibustion on knee joint dysfunction after anterior cruciate ligament reconstruction," Traditional Chinese medicine, vol. 27, no. 4, pp. 20-21, 2018. 
[24] J. X. Li and Z. Q. Huang, "Traditional Chinese medicine internal and external usage of early rehabilitation after ACL reconstruction under arthroscopy," Journal of Chang China Medical University, vol. 33, no. 2, pp. 292-295, 2017.

[25] Y. Liang, S. M. Zhang, Y. Hu, H. W. Zhang, and M. Li, “The intermediate stage study of traditional Chinese medicine combing rehabilitation training in postoperative ACL construction under arthroscopy," Chinese Journal of Traditional Medicine of Orthopaedics \& Traumatology:, vol. 33, no. 2, pp. 292-295, 2010.

[26] Y. Liang, S. M. Zhang, Y. Hu, H. W. Zhang, and R. X. Yuan, "Effects of traditional Chinese medicine and rehabilitation training on knee joint function after anterior cruciate ligament reconstruction in arthroscopy," Chinese Journal of Clinical Rehabilitation, vol. 10, no. 27, pp. 6-10, 2006.

[27] Z. P. Tang, Y. J. Li, and S. H. Li, "The influence of integrative medicine rehabilitation therapy on proprioceptive sense recovery of the patients after the surgery of knee anterior cruciate ligament reconstruction," West Journal of Traditional Chinese Medicine, vol. 30, no. 4, pp. 105-107, 2017.

[28] C. Y. Wang, The Effects of Postoperative Oral Administration of Traditional Chinese Medicine and Intraoperative Retention of the Tibia Stump on the Healing of the Tibia after Anterior Cruciate Ligament Reconstruction, Liaoning University of Traditional Chinese Medicine, Shenyang, China, 2019.

[29] H. Wang, E. R. Zhang, and S. Z. Huang, "Experience of taohong siwu decoction in treating early rehabilitation of anterior cruciate ligament reconstruction," Chinese Medicine of MOD Distance Education China, vol. 17, no. 8, pp. 48-49, 2019.

[30] J. Wang, "Effect of acupoint needling and rehabilitation training on recovery and prognosis of knee joint function after anterior cruciate ligament reconstruction," Journal of New Chinese Medicine, vol. 52, no. 8, pp. 145-147, 2020.

[31] J. Velázquez-Saornil, B. Ruíz-Ruíz, D. Rodríguez-Sanz, C. Romero-Morales, D. López-López, and C. Calvo-Lobo, "Efficacy of quadriceps vastus medialis dry needling in a rehabilitation protocol after surgical reconstruction of complete anterior cruciate ligament rupture," Medicine (Baltimore), vol. 96, no. 17, pp. 1-10, 2017.

[32] D. E. Meuffels, M. T. Poldervaart, R. L. Diercks et al., "Guideline on anterior cruciate ligament injury," Acta Orthopaedica, vol. 83, no. 4, pp. 379-386, 2012.

[33] G. Samitier, A. I. Marcano, E. Alentorn-Geli, R. Cugat, K. W. Farmer, and M. W. Moser, "Failure of anterior cruciate ligament reconstruction," The archives of bone and joint surgery, vol. 3, no. 4, pp. 220-240, 2015.

[34] F. Sepúlveda, L. Sánchez, E. Amy, and W. Micheo, “Anterior cruciate ligament injury," Current Sports Medicine Reports, vol. 16, no. 3, pp. 172-178, 2017.

[35] K. D. Shelbourne and T. Gray, "Minimum 10-year results after anterior cruciate ligament reconstruction," The American Journal of Sports Medicine, vol. 37, no. 3, pp. 471-480, 2009.

[36] D. Adams, D. Logerstedt, A. Hunter-Giordano, M. J. Axe, and L. Snyder-Mackler, "Current concepts for anterior cruciate ligament reconstruction: a criterion-based rehabilitation progression," Journal of Orthopaedic \& Sports Physical Therapy, vol. 42, no. 7, pp. 601-614, 2012.

[37] V. Kandhari, T. D. Vieira, H. Ouanezar et al., "Clinical outcomes of arthroscopic primary anterior cruciate ligament repair: a systematic review from the scientific anterior cruciate ligament network international study group," Arthroscopy: The Journal of Arthroscopic \& Related Surgery, vol. 36, no. 2, pp. 594-612, 2020.
[38] D. Mouarbes, J. Menetrey, V. Marot, L. Courtot, E. Berard, and E. Cavaignac, "Anterior cruciate ligament reconstruction: a systematic review and meta-analysis of outcomes for quadriceps tendon autograft versus bone-patellar tendonbone and hamstring-tendon autografts," The American Journal of Sports Medicine, vol. 47, no. 14, pp. 3531-3540, 2019.

[39] H. J. Ha and M.-S. Oh, "A systemic review of Korean medical treatment combined with continuous passive motion therapy on the knee joint postoperative rehabilitation," Journal of Korean Medicine Rehabilitation, vol. 27, no. 3, pp. 33-45, 2017.

[40] D. J. Crespin, K. H. Griffin, J. R. Johnson et al., “Acupuncture provides short-term pain relief for patients in a total joint replacement program," Pain Medicine, vol. 16, no. 6, pp. 1195-1203, 2015.

[41] I. Park, C. Jung, and M. Jang, "A randomized clinical trial of local acupoints compared with distal acupoints in degenerative osteoarthritis on knee," Journal of Korean Acupunct and Moxibustion Society, vol. 25, no. 2, pp. 227-242, 2008.

[42] F. J. Zijlstra, I. Van Den Berg-De Lange, F. J. P. M. Huygen, and J. Klein, "Anti-inflammatory actions of acupuncture," Mediators of Inflammation, vol. 12, no. 2, pp. 59-69, 2003.

[43] B. Kavoussi and B. E. Ross, "The neuroimmune basis of antiinflammatory acupuncture," Integrative Cancer Therapies, vol. 6, no. 3, pp. 251-257, 2007.

[44] J. Filshie, A. White, and M. Cummings, Medical Acupuncture; A Western Scientific Approach, Elsevier, Amsterdam, Netherlands, 2nd edition, 2016.

[45] S. Ortega-Cebrian, N. Luchini, and R. Whiteley, "Dry needling: effects on activation and passive mechanical properties of the quadriceps, pain and range during late stage rehabilitation of ACL reconstructed patients," Physical Therapy in Sport, vol. 21, pp. 57-62, 2016.

[46] Y. Mikashima, T. Takagi, T. Tomatsu, M. Horikoshi, K. Ikari, and S. Momohara, "Efficacy of acupuncture during post-acute phase of rehabilitation after total knee arthroplasty," Journal of Traditional Chinese Medicine, vol. 32, no. 4, pp. 545-548, 2012.

[47] W. Zhang, S. Wang, R. Zhang et al., "Evidence of Chinese herbal medicine Duhuo Jisheng decoction for knee osteoarthritis: a systematic review of randomised clinical trials," $B M J$ Open, vol. 6, no. 1, p. e008973, 2016.

[48] B. Chen, H. Zhan, J. Marszalek et al., "Traditional Chinese medications for knee osteoarthritis pain: a meta-analysis of randomized controlled trials," The American Journal of Chinese Medicine, vol. 44, no. 04, pp. 677-703, 2016.

[49] S. Zhu, Y. Song, X. Chen, and W. Qian, "Traditional Chinese and western medicine for the prevention of deep venous thrombosis after lower extremity orthopedic surgery: a metaanalysis of randomized controlled trials," Journal of Orthopaedic Surgery and Research, vol. 13, no. 1, pp. 72-79, 2018.

[50] H.-S. Han, "Anti-inflammatory effect of Angelicae Gigantis radix water extract on LPS-stimulated mouse macrophages," The Korea Journal of Herbology, vol. 28, no. 5, pp. 113-119, 2013.

[51] X. Bi, L. Han, T. Qu et al., "Anti-inflammatory effects, SAR, and action mechanism of monoterpenoids from radix Paeoniae alba on LPS-stimulated RAW 264.7 cells," Molecules, vol. 22, no. 5, pp. 715-719, 2017.

[52] J. Wu, J. Li, W. Li et al., "Achyranthis bidentatae radix enhanced articular distribution and anti-inflammatory effect of berberine in Sanmiao Wan using an acute gouty arthritis rat 
model," Journal of Ethnopharmacology, vol. 221, pp. 100-108, 2018.

[53] L. Zhang, Y.-X. Cheng, A.-L. Liu, H.-D. Wang, Y.-L. Wang, and G.-H. Du, "Antioxidant, anti-inflammatory and antiinfluenza properties of components from chaenomeles speciosa," Molecules, vol. 15, no. 11, pp. 8507-8517, 2010.

[54] Q. Chen and W. Wei, "Effects and mechanisms of glucosides of chaenomeles speciosa on collagen-induced arthritis in rats," International Immunopharmacology, vol. 3, no. 4, pp. 593-608, 2003.

[55] N. J. Collins, D. Misra, D. T. Felson, K. M. Crossley, and E. M. Roos, "Measures of knee function: international knee documentation committee (IKDC) subjective knee evaluation form, knee injury and osteoarthritis outcome score (KOOS), knee injury and osteoarthritis outcome score physical function short form (KOOS-PS), knee ou," Arthritis Care \& Research, vol. 63, no. S11, pp. S208-S228, 2011.

[56] M. W. C. Chan, X. Y. Wu, J. C. Y. Wu, S. Y. S. Wong, and V. C. H. Chung, "Safety of acupuncture: overview of systematic reviews," Scientific Reports, vol. 7, no. 1, pp. 1-11, 2017.

[57] S. Xu, L. Wang, E. Cooper et al., "Adverse events of acupuncture: a systematic review of case reports," Evidence-based Complementary and Alternative Medicine, vol. 2013, no. 1, pp. 1-15, 2013.

[58] S. Y. Kim, J. E. Park, H. J. Seo et al., "NECA's guidance for undertaking systematic reviews and meta-analyses for intervention," Seoul Natl Evidence-based Healthc Collab Agency, vol. $25,2011$. 